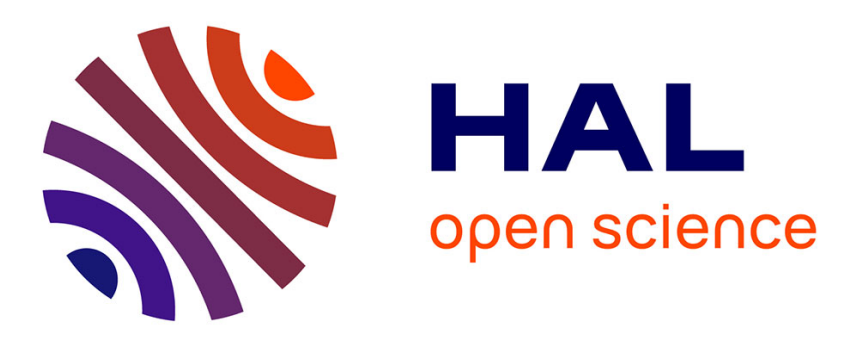

\title{
Low doses of triazine xenobiotics mobilize ABA and cytokinin regulations in a stress- and low-energy-dependent manner
}

Diana Alberto, Ivan Couée, Stéphanie Pateyron, Cécile Sulmon, Gwenola Gouesbet

\section{To cite this version:}

Diana Alberto, Ivan Couée, Stéphanie Pateyron, Cécile Sulmon, Gwenola Gouesbet. Low doses of triazine xenobiotics mobilize ABA and cytokinin regulations in a stress- and low-energy-dependent manner. Plant Science, 2018, 274, pp.8-22. 10.1016/j.plantsci.2018.04.025 . hal-01862321v2

\section{HAL Id: hal-01862321}

https://hal-univ-rennes1.archives-ouvertes.fr/hal-01862321v2

Submitted on 14 Sep 2018

HAL is a multi-disciplinary open access archive for the deposit and dissemination of scientific research documents, whether they are published or not. The documents may come from teaching and research institutions in France or abroad, or from public or private research centers.
L'archive ouverte pluridisciplinaire HAL, est destinée au dépôt et à la diffusion de documents scientifiques de niveau recherche, publiés ou non, émanant des établissements d'enseignement et de recherche français ou étrangers, des laboratoires publics ou privés. 


\section{Low doses of triazine xenobiotics mobilize ABA and cytokinin regulations in a stress- and low-energy-dependent manner}

Authors: Diana Alberto ${ }^{1}$, Ivan Couée ${ }^{1}$, Stéphanie Pateyron ${ }^{2,3}$, Cécile Sulmon ${ }^{1}$, Gwenola Gouesbet $^{1 *}$

\section{Affiliation:}

1. Université de Rennes 1 / Centre National de la Recherche Scientifique, UMR 6553 ECOBIO, F-35000 Rennes, France

2. Institute of Plant Sciences Paris Saclay IPS2, CNRS, INRA, Université Paris-Sud, Université Evry, Université Paris-Saclay, Orsay, France

3. Institute of Plant Sciences Paris-Saclay IPS2, Paris Diderot, Sorbonne Paris-Cité, Orsay, France

\section{Correspondence:}

Gwenola Gouesbet

gwenola.gouesbet@univ-rennes1.fr

Number of figures: 4

Number of tables: 5

Number of supplemental data: 8

\section{Highlight}

- Atrazine and its degradation products induce changes in gene expression 
- Low doses of triazines can act through canonical-target-independent regulations

- $\mathrm{ABA}-$ and CK-regulated processes are involved in triazine responses

- Stress-related signaling is implicated in the perception of low doses of triazines

\begin{abstract}
The extent of residual contaminations of pesticides through drift, run-off and leaching is a potential threat to non-target plant communities. Arabidopsis thaliana responds to low doses of the herbicide atrazine, and of its degradation products, desethylatrazine and hydroxyatrazine, not only in the long term, but also under conditions of short-term exposure. In order to investigate underlying molecular mechanisms of low-dose responses and to decipher commonalities and specificities between different chemical treatments, parallel transcriptomic studies of the early effects of the atrazine-desethylatrazine-hydroxyatrazine chemical series were undertaken using whole-genome microarrays. All of the triazines under study produced coordinated and specific changes in gene expression. Hydroxyatrazine-responsive genes were mainly linked to root development, whereas atrazine and desethylatrazine mostly affected molecular signaling networks implicated in stress and hormone responses. Analysis of signaling-related genes, promoter sites and shared-function interaction networks highlighted the involvement of energy-, stress-, abscisic acid- and cytokinin-regulated processes, and emphasized the importance of cold-, heat- and drought-related signaling in the perception of low doses of triazines. These links between low-dose xenobiotic impacts and stress-hormone crosstalk pathways give novel insights into plant-pesticide interactions and plant-pollution interactions that are essential for toxicity evaluation in the context of environmental risk assessment.
\end{abstract}

Keywords: chemical stress, signaling, pesticide degradation products, transcription factors, transcriptomics, environmental pollution

\title{
1. Introduction
}


Conventional agriculture requires significant crop protection in order to reduce pest-induced yield losses. In the last fifty years, hundreds of molecules, of both natural and anthropogenic origins, have been released in environment, specifically targeting vital mechanisms in weeds, arthropods and microorganisms. Such widespread application has led to global diffusion of xenobiotic pesticides in soil and water compartments [1]. Besides desired action on pests, pesticides, such as herbicides, affect non-target organisms in agricultural areas [2]. Pesticides, and particularly herbicides, have different degrees of persistence in environmental compartments according to chemical structure and to physicochemical properties of environmental matrices [3]. Soil and water detection of herbicide-related molecules indicates that spontaneous chemical reactions, microorganism-related and plant-related processes [4] cause the liberation of degradation products, whose impacts on ecosystem balance are still poorly understood.

Atrazine (ATZ) is a selective and systemic herbicide used to control broadleaf weeds. Because of widespread use and persistence, atrazine has emerged as a pollutant of environmental concern [5]. It is currently applied in most regions of America, Asia and Oceania. Moreover, even several years after end of use, as in the European Union where it was banned in 2004, it is still detected in the environment [6]. ATZ targets D1 subunit of Photosystem II (PSII), thus leading to interruption of the photosynthetic electron transport chain, to loss of PSII efficiency, and to production of Reactive Oxygen Species (ROS) [7].

Triazines pollution in the environment involves not only ATZ itself, but also chemically-similar compounds derived from ATZ degradation. Among them, desethylatrazine (DEA) and hydroxyatrazine (HA) are frequently detected in water streams [5]. Little information is available about their effects on plants. Serra et al. [8] demonstrated that realistic environmental concentrations of HA did not affect Arabidopsis photosynthesis and growth, but induced cryptic effects modifying plant metabolism and gene expression. This apparently non-phytotoxic degradation product can therefore act on unknown molecular targets. Alberto et al. [5] recently showed that, under conditions of transient root exposure ( $24 \mathrm{~h}$ of exposure) to low doses of ATZ, DEA and HA, Arabidopsis responded to triazines with significant effects on growth and development in dose-dependent and differential manners. ATZ effects ranged from major impact on night-time respiration at low dose $(1 \mu \mathrm{M})$ to multiple impacts on respiration, photosynthesis, root growth and plant growth at higher dose $(10 \mu \mathrm{M})$. This significant impact on night-time respiration indicated that lower doses of ATZ were not innocuous for plants, even when PSII efficiency was not affected. DEA exposure resulted in significant effects on root 
growth, non-photochemical quenching (NPQ) and night-time respiration at high dose $(10 \mu \mathrm{M})$. In contrast, HA caused minor and transitory physiological effects on root growth, thus highlighting the key role of chloride moiety, present in ATZ and DEA, but not in HA, for triazine toxicity. These discrepancies of effects indicate complex interactions with non-PSII targets. ATZ and DEA may affect physiological processes through respiratory and mitochondrial impacts in addition to PSII perturbation at higher levels. However, DEA also interfered with root growth independently from PSII efficiency inhibition and HA may act on root meristem activity through an alternative action mode. Such complex interactions have also been revealed in the context of sugar-induced protection against ATZ exposure [9], where ATZ did not affect photosynthesis in the short-term, but induced rapid and important gene expression variations, thus indicating underlying regulation and signaling mechanisms that remained to be characterized.

In order to determine early regulatory processes and signaling pathways involved in the action of ATZ, DEA and HA, a whole-genome transcriptomic approach was undertaken in Arabidopsis under conditions of short-term (24 h of exposure), root-level exposure to $1 \mu \mathrm{M}$ ATZ, DEA or HA, reflecting environmental levels of diffuse or transient pollution. This genome-wide approach revealed specific signatures and common responses. Detailed analysis of these patterns highlighted potential disruption of energy, carbon and nitrogen homeostasis, and potential activation of cell-wall and defense processes. The significant proportion of transcription- and signaling-related genes in the sets of differentially-expressed genes provide novel insights into the primary signaling components that are early affected by triazines in the course of plant-xenobiotic interactions.

\section{Material and Methods}

\subsection{Plant material and growth conditions}

Seedlings of Arabidopsis thaliana (ecotype Columbia, Col0) were cultivated after seed sterilization as described in Alberto et al. [5]. RNA extractions for microarray and qRT-PCR experiments were performed on thirteen-d-old plantlets transferred at 1.04 developmental growth stage [10] on control or to triazine [1 $\mu \mathrm{M}$ ATZ, DEA or HA (Sigma, St. Louis, MO, USA)] $0.8 \%$ agar-Hoagland media during $24 \mathrm{~h}$. Each treatment was repeated in order to obtain independent biological and experimental replicates. In each replicates, 60 plantlets consisting in the pool of 3 distinct sets of 10 to 15 individual plants treated as described above and 
corresponding to a given treatment were harvested, frozen in liquid nitrogen and extracted for RNA.

\subsection{Transcriptome profiling microarray analysis}

Microarray analysis was carried out at the Institut of Plant Sciences Paris-Saclay (IPS2, Orsay, France), using the CATMAv7 [11] based on AGILENT technology on the Transcriptomic Plateform POPS (transcriptOmic Platform of iPS2). The CATMAv7 design of Arabidopsis thaliana genome have been made with gene annotations include in FLAGdb++, an integrative database around plant genome (http://urgv.evry.inra.fr/FLAGdb). The single high density CATMAv7 microarray slide contains four chambers, each containing 149916 primers. Each $60 \mathrm{bp}$ primer is triplicate in each chamber for robust analysis and in both strand. As part of all probes, 35754 in triplicate correspond to gene TAIRv8 (among which 476 probes corresponding to mitochondrial and chloroplast genes) +1289 probes corresponding to EUGENE software predictions +658 probes for miRNA/MIR, and finally 240 controls. For each condition, RNA extractions for microarray analysis were performed on two independent biological and experimental replicates. Total RNA for each replicate was extracted from 60 thirteen d-old plantlets transferred at 1.04 developmental growth stages [10] on four different treatment media during $24 \mathrm{~h}$ as indicated above using QIAGEN RNeasy Plant Mini Kit, according to the supplier's instructions. For each comparison, two technical replicates with fluorochrome reversal were performed for each biological replicate (i.e. four hybridizations per comparison). The labeling of cRNAs with Cy3-dUTP or Cy5-dUTP was performed as described in Two-Color Microarray-Based Gene Expression Analysis Low Input Quick Amp Labeling manual (@) Agilent Technologies, Inc.). The hybridization and washing were performed according to Agilent Microarray Hybridization Chamber User Guide instructions () Agilent Technologies, Inc.). Two micron scanning was performed with InnoScan900 scanner (Innopsys $^{\mathrm{R}}$, Carbonne, FRANCE) and raw data were extracted using Mapix ${ }^{\mathrm{R}}$ software (Innopsys $^{\mathrm{R}}$, Carbonne, FRANCE). ATZ, DEA and HA conditions were compared to the control.

\subsection{Statistical analysis of microarray data}

Experiments were designed with the statistics group of the IPS2. For each array, the raw data comprised the logarithm of median feature pixel intensity at wavelengths $635 \mathrm{~nm}$ (red) and 532 $\mathrm{nm}$ (green). For each array, a global intensity-dependent normalization using the loess 
procedure [12] was performed to correct the dye bias. The differential analysis is based on the log-ratios averaging over the duplicate probes and over the technical replicates. Hence the numbers of available data for each gene equals the number of biological replicates and are used to calculate the moderated t-test [13]. Under, the null hypothesis, no evidence that the specific variances vary between probes is highlighted by Limma and consequently the moderated tstatistic is assumed to follow a standard normal distribution.

To control the false discovery rate, adjusted p-values found using the optimized False Discovery Rate (FDR) approach of Storey and Tibshirani [14] are calculated. We considered as being differentially expressed the probes with an adjusted $p$-value $\leq 0.05$. Analysis was done with the $\mathrm{R}$ software [15]. The function SqueezeVar of the library Limma has been used to smooth the specific variances by computing empirical Bayes posterior means. The library kerfdr has been used to calculate the adjusted p-values.

\subsection{Analysis of microarray data}

Hierarchical clustering was performed using Cluster 3.0 software (http://bonsai.hgc.jp/ mdehoon/software/cluster) and Java Tree View (http://jtreeview.sourceforge.net). GO annotation retrieval and GO Slim Classification developed at TAIR (http://www.arabidopsis.org/tools/bulk/go/index.jsp) were used to retrieve GO and GO Slim terms associated with differentially expressed genes (DEGs). Overrepresented and under-represented functional categories were determined by Classification SuperViewer (http://bar.utoronto.ca/) with normalized class score option. Putative sharedfunction and protein-protein interaction networks were determined by mapping DEGs onto STRING v.10 global Arabidopsis networks [16].

Promoter search for cis-elements was undertaken with Arabidopsis promoter motif search programs using $2 \mathrm{~kb}$ upstream regions from translation start sites (http://stan.cropsci.uiuc.edu/cgi-bin/elefinder/compare.cgi). Overrepresentation of octameric sequences derived from promoter consensus sequence $(A C T)(A G)(G T) A T(A C T)(C T)(A C G T)$ was estimated as described by Brenner and Schmülling [17]. Motifs in promoters were counted using Promomer (http://bbc.botany.utoronto.ca/ntools/cgi-bin/BAR_Promomer.cgi) and significantly enriched motifs for DEG promoters were calculated against a control set of least likely CK-induced genes [17]. 


\subsection{Complementary analysis of transcriptome profiling datasets}

Gene expression data registered as E-MEXP-411 were extracted from Array-Express (http://www.ebi.ac.uk/arrayexpress/). Analysis of high-dose ATZ effects on gene expression was derived from Ma (10 $\mu \mathrm{M}$ ATZ in the presence of $80 \mathrm{mM}$ mannitol as osmotic control) and $\mathrm{Sa}(10 \mu \mathrm{M}$ ATZ in the presence of $80 \mathrm{mM}$ sucrose) comparisons.

\section{6 qRT-PCR analysis}

For each condition, qRT-PCR experiments were carried out using RNA from three independent biological and experimental replicates. Total RNA for each replicate was extracted from 60 thirteen d-old plantlets transferred at 1.04 developmental growth stages [10] on four different treatment media during $24 \mathrm{~h}$ as indicated above. Synthesis of cDNAs was carried out using Iscript $^{\mathrm{TM}}$ cDNA Synthesis kit (BioRad, Hercules, CA, USA). Resulting cDNAs were used to determine expression profiles. Quantitative PCR was performed using LightCycler $^{\circledR} 480$ SYBR green I master (Roche Diagnostics, Meylan, France). Conditions were as follows: $95^{\circ} \mathrm{C} 3 \mathrm{~min}$, and $40\left(95^{\circ} \mathrm{C} 15 \mathrm{~s}, 60^{\circ} \mathrm{C} 30 \mathrm{~s}, 72^{\circ} \mathrm{C} 30 \mathrm{~s}\right)$ cycles. All reactions were performed in duplicates (two technical replicates). Specific primers for each gene were designed using Primer3Plus software (Supplemental Table 1). Gene expression was quantified relatively to $P P 2 A$ housekeeping gene expression by using LightCycler® 480 software.

\subsection{Data Deposition}

Microarray data were deposited at Gene Expression Omnibus (GEO) (http://www.ncbi.nlm.nih.gov/geo/; accession number GSE88788) and at CATdb [18] (http://urgv.evry.inra.fr/CATdb/; Project: AU15-04_Xeno) according to Minimum Information About a Microarray Experiment (MIAME) standards.

\section{Results and discussion}

3.1 Short-term exposure to low doses of triazine xenobiotics triggers significant gene expression dynamics 
To investigate the molecular effects of triazines at low doses and to decipher their commonalities and specificities, a transcriptomic approach using CATMA arrays was undertaken. Arabidopsis plantlets were exposed to $1 \mu \mathrm{M}$ ATZ, DEA, or HA during $24 \mathrm{~h}$. Shortterm transfer exposure to $1 \mu \mathrm{M}$ triazine induces no observable adverse effect on root growth rate for ATZ and DEA and on PSII efficiency for DEA and HA [5]. HA slightly decreases (approximately 15\% decrease) root growth and ATZ slightly decrease (approximately 10\% decrease) PSII efficiency [5]. A total of 198 genes was differentially expressed (p-value $\leq 0.05$ after Bonferroni correction and $\mid \log _{2}($ ratio) $\mid>1)$ in at least one of the 3 comparisons (Supplemental Table 2). Relatively to control, ATZ treatment caused differential expression of 83 genes (67 induced, 16 repressed). DEA treatment resulted in 115 differentially expressed genes (DEGs) (89 induced, 26 repressed), whereas HA changed the expression of 89 genes (80 induced, 9 repressed). No miRNAs showed any significant modification of expression (data not shown). These chemical treatments under conditions of low toxicity and short-term exposure therefore revealed a small, but significant, number of DEGs. Low doses of ATZ and of its metabolites had therefore the potential to affect transcript levels by modifying transcription or turnover of mRNA.

Distribution and overlap of transcript modifications were represented by Venn diagrams (Figure 1), composition of each group in diagrams is illustrated in Supplemental Figure 1. Most DEGs resulted from upregulations rather than downregulations. DEA and HA specifically induced more genes (42) than ATZ (18). More genes were commonly induced or repressed between ATZ and DEA (16 and 3 respectively) than between ATZ and HA (7 and 1 respectively), or DEA and HA (5 and 1 respectively). Almost 21 genes were specifically repressed by DEA, while ATZ and HA respectively downregulated 11 and 6 genes. Finally, only 26 DEGs and 1 DEG were, respectively, induced and repressed by all treatments, representing $13 \%$ of DEGs. Thus, ATZ induced less modification of expression than its metabolites, whereas it was the most effective triazine for modifying physiological parameters at higher doses or under longterm exposure [5]. Transcript-level modifications could therefore be ascribed to xenobiotic chemical structure rather than to physiological impact.

Relationships between triazine treatments were determined by two-dimensional hierarchical cluster analysis by average linkage using Cluster 3.0. The heatmap showing the 198 DEGs (Figure 2) clearly pointed out that responses to ATZ and DEA, which both carry a chloride moiety, were closer than those observed after HA treatment. The fact that ATZ, DEA and HA treatments individually caused specific induction or repression of gene expression indicated 
that Arabidopsis could not readily metabolize ATZ herbicide into DEA and HA degradation products. Otherwise, molecular responses to triazines would have shown much greater overlaps.

The transcriptomic approach therefore demonstrated that short xenobiotic exposure under conditions of no or low toxicity [5] can result in significant perturbations of gene expression. In agreement with their closer chemical structure, ATZ and DEA presented important common features of transcriptomic responses, contrasting with HA-specific responses (Figures 1-2). Yang et al. (2014) [19] have shown that plants, during short-term stress, give priority to pathways enabling survival, whereas, for long-term stress, pathways leading to growth are preferred, although over-growth is prevented to save energy. Hormone signaling during these different types of stress promotes survival or growth with effects on gene expression or on corresponding proteins. ATZ and DEA show similar long-term effects on plant growth with however very different time courses of impact [5]. The differences of regulatory effects between ATZ and DEA may contribute to these differences in chronological impact

\section{Coordinated changes of cellular and molecular functions}

Concise functional classification of DEGs was established using Gene Ontology (GO) Slim Classification. GO Slim for $\quad$ Classification (http://www.arabidopsis.org/tools/bulk/go/index.jsp) from the Arabidopsis Information Resource (TAIR) gives functional categorization of GO Slim terms in each keyword category (biological process, molecular function, cellular component). Induced and repressed GO Slim terms were counted according to each treatment (Supplemental Figure 2). Enrichment, determined by Classification SuperViewer, was analyzed by assigning a normalized frequency to each GO Slim term for each treatment (Table 1). Supplemental Table 3 gives for each gene set (induced or repressed genes according to each triazine treatment) classical GO annotation (GO term) associated to each locus and corresponding broader category-termed GO Slim. Categorization of DEGs according to treatments showed, that, although treatments participated in common general effects, ATZ and DEA produced closer effects (Figure 2). Both treatments 
induced genes related to "response to abiotic or biotic stimulus", and more particularly to GO Slim term "response to stress", which was significantly overrepresented (Table 1). This latter category was also overrepresented among repressed genes for the three triazines (Supplemental Figure 2A, Table 1). Only HA produced overrepresentation of induced genes linked to “developmental processes". Several GO terms grouped under GO Slim term "other biological processes" were overrepresented among genes induced or repressed by triazines. According to Supplemental Table 3, most of these GO terms were linked to hormonal responses. Under GO Slim term "other cellular processes", which was overrepresented in induced genes, most of related GO terms were linked to metabolism or catabolism processes, to regulation of transcription for ATZ- and DEA-induced genes, or to growth and root differentiation for HAinduced genes. Molecular functions linked to "transcription factor (TF) activity" were also highly and significantly induced by ATZ and DEA. The transcriptomic profile induced by HA was therefore characterized by responsive genes linked to development and root differentiation, while ATZ and DEA preferentially affected processes and functions related to transcription regulation and stress responses.

All treatments significantly induced loci carrying "hydrolase activity" (Supplemental Figure 2B, Table 1). GO Slim term "other enzyme activity", which was significantly overrepresented in triazine-induced genes and in DEA-repressed genes, consisted in GO terms mostly linked to oxidoreductase, and more particularly peroxidase, activities. Cellular localization of over- and under-represented functions and processes were linked to "extracellular part" category, especially in the case of HA. ATZ and HA treatments were responsible for overrepresentation of genes related to "cell wall" (Supplemental Figure 2C, Table 1). Finally, functions and processes linked to "mitochondria" and "plasma membrane" were underrepresented after, respectively, HA and ATZ/DEA treatments.

Functional classification of triazine-sensitive genes emphasized important common responses to all triazines that were linked to hormone, metabolism and stress. Such impacts under conditions of low doses were reminiscent of previously-described transcriptomic responses of plants to xenobiotics [8,9,20-23]. Moreover, detoxifying mechanisms, such as those related to peroxidase activities, are part of primary responses to xenobiotics in plants [20]. However, overrepresented cellular localizations like "cell wall" and "extracellular" components (Table 1) gave novel insights into the potential importance of cell wall and intercellular signal transduction for triggering and regulating plant-triazine interactions.

\subsection{Interference with carbon and energy sensing}


Tables 2 and 3 describe expression patterns of typical triazine-responsive DEGs derived from CATMA analysis (Table 2) and RT-qPCR individual gene analysis (Table 3A). Table 2 also gives expression patterns under conditions of high ATZ stress (Ma, mannitol $80 \mathrm{mM}$ plus atrazine $10 \mu \mathrm{M}$ ) and of ATZ tolerance (Sa, sucrose $80 \mathrm{mM}$ plus atrazine $10 \mu \mathrm{M}$ ) where sucrose induces protection against ATZ impact [9]. The most highly-induced genes under conditions of low doses of triazines included AT3G19430, AT5G54370 and AT5G60530, which encode Late Embryogenesis Abundant (LEA)-related proteins (Tables 2-3A). LEA proteins are ABAresponsive, induced by stress and senescence conditions and important for desiccation and heat protection [24,25]. In contrast, these genes were not induced by high ATZ doses in absence or presence of sucrose (Table 2), thus indicating the specificity of low-dose effects.

All triazines highly increased expression of genes linked to glucosinolate catabolism. BGLU34, $B G L U 35$ and BGLU36, which were in part responsible for overrepresentation of "hydrolase" GO term (Table 1), encode Beta-glucosidases with myrosinase activity involved in glucosinolate catabolism. Genes encoding mannose-binding jacalin-related lectin superfamily proteins (AT1G52050, AT1G52060) known to interact with myrosinases, were highly induced by low doses of triazines (Tables 2-3A). Such jacalin-related lectins, which are inducible by stress hormones [salicylic acid (SA), jasmonic acid (JA), abscisic acid (ABA)], are associated with plant adaptation to stressful environments and stress signal transduction [26]. Glucosinolates are nitrogen- and sulfur-containing compounds, whose catabolism produce toxic compounds, such as nitriles and thiocyanates, involved in plant defense [27,28]. Glucosinolate metabolism is thus linked to nitrile detoxication involving nitrilases that catalyze transformation of nitrile to ammonia [27,29]. Interestingly, nitrilase-encoding NIT2 gene was induced by DEA and by $10 \mu \mathrm{M}$ ATZ in absence or presence of sucrose (Table 2). NIT2, which is involved in auxin biosynthesis [30], was also upregulated at protein level in response to ABA [29]. In contrast, genes linked to glucosinolate biosynthesis [28] and positively controlled by Myb TFs were downregulated. $A O P 2$, which encodes a 2-oxoglutarate-dependent dioxygenase, and GSTU20 and GSTF12, both encoding glutathione transferases, were repressed by DEA and by high doses of ATZ whether in absence or presence of sucrose (Table 2).

Triazines regulated several genes of energy metabolism and low-energy responses. BCAT2, encoding a chloroplast branched-chain amino acid (BCAA) aminotransferase [31], and $A T 1 G 19530$, encoding a protein of unknown function involved in low-oxygen stress response [32], were induced by DEA and repressed by HA (Table 2). They also showed high level of induction by high doses of ATZ, whether in absence or presence of sucrose. Moreover, the 
slight trend of induction by $1 \mu \mathrm{M}$ ATZ in microarray analysis (data not shown) was confirmed by individual gene analysis in the case of BCAT2 (Table 3A). BCAT2 is involved in BCAA (valine, leucine and isoleucine) catabolism leading to, inter alia, glutamate, metabolites feeding the tricarboxylic acid cycle under energy-limited conditions, and respiratory substrates $[31,33]$. BCAA catabolism contributes to maintain amino acid and cell homeostasis during low energy stress [31,33,34]. Pires et al. [35] demonstrated that, via mitochondrial alternative pathways of respiration, BCAA catabolism also plays an important role in tolerance mechanisms during drought stress. Low energy stress leads to asparagine biosynthesis through the induction of ASN1 gene encoding glutamine-dependent asparagine synthetase [36]. This gene was induced by low doses of ATZ and DEA and by high doses of ATZ in absence or presence of sugar (Tables 2, 3A). ASN1 and DIN1, a senescence-associated gene induced by DEA (Table 2), are induced by conditions of photosynthesis and respiration limitation, such as darkness, PSIIinhibiting DCMU herbicide treatment, and hypoxia [37]. Moreover, correlated inductions of $A S N 1$ and BCAT2 have been already described during low-energy stress, such as dark treatment [31]. All of these sugar-repressed loci are regulated by Sucrose-non-fermenting-1 (SNF1)related protein kinase1 (SnRK1), an energy depletion sensor protein playing key roles in plant growth and development and in the regulation of specific stress-inducible genes involved in physiological adaptation to stress [38]. Induction of these sugar-repressed and low-energyinducible genes seemed to be a general feature of triazine action, at low or high doses, with opposite effect of HA on BCAT2 expression (Table 2), thus highlighting the chemical specificity (ATZ, DEA) of such action. Moreover, ATZ induction of sugar-repressed genes even in presence of sucrose pointed out that ATZ acted through regulatory processes rather than through direct metabolic effects [34]. Gutiérrez et al. [39] demonstrated that CIRCADIAN CLOCK ASSOCIATED1 (CCA1) TF, a central component of the circadian clock, controls Nassimilation in plants through its own regulation by organic $\mathrm{N}$ and through direct regulation of both ASN1 and NRT2.6. Co-expression of these genes under conditions of triazine treatment may therefore reflect interferences between triazine responses and circadian clock pathways. Moreover, the SnRK1 energy sensor and the circadian clock interact with each other [40].

Impact of low doses of triazines on carbon, nitrogen and energy processes was also reflected in responses of genes encoding light-harvesting chlorophyll a/b-binding (LHC) proteins that constitute the antenna system of photosynthetic apparatus (Table 2). LHCB3 and LHCA4 were generally induced by low doses of triazines despite absence of effect or weak effect on PSII efficiency [5]. In contrast, high doses of ATZ had repressive effects on LHCB3 and LHCA4 
photosynthesis-related genes, thus again emphasizing the specificity of low-dose responses (Table 2). Negative or positive impacts of low doses of triazines on photosystem-related gene expression occurred in absence of significant interference with PSII canonical target of ATZ, and may thus be related to same mechanisms of energy sensing as those leading to regulation of ASN1, DIN1, BCAT2 and At1g19530 expression.

Structurally-diverse xenobiotics can cause perturbations of energy, carbon, mitochondrial and nitrogen homeostasis $[4,7,41,42]$. The present results comparing ATZ and DEA establish that coordinated regulation of energy, $\mathrm{C}$ and $\mathrm{N}$ metabolisms by low doses of xenobiotics can occur prior to any significant physiological or biochemical perturbation. This strongly suggests that triazines, applied at low doses, act independently of ATZ canonical target, and may interact with signaling targets, such as energy sensors (SnRK1), that can allow plants to sense threats that potentially endanger metabolic homeostasis. The AMP-activated kinase (AMPK) energy sensor of mammalian cells, which belongs to the same eukaryotic protein kinase family as SnRK1, plays such a role. It responds to stimuli, including xenobiotics, that threaten to decrease ATP levels, and induces coordinated transcriptomic responses decreasing energy consuming pathways and increasing catabolism [43].

\subsection{Triazine-regulated transcriptional networks}

Coordinated modification of functional categories linked to stress responses (Table 1) and of metabolic genes expression (Table 2) suggested the involvement of regulation processes in response to triazines. Among the 198 triazine-responsive DEGs, a significant proportion $(15.65 \%, 31$ genes) was related to regulation of expression and signaling processes (Table 4). Among these 31 genes, only 4 were differentially expressed in response to HA. These 4 genes were involved in the developmental processes related to root and hormonal context, in agreement with developmental effects of low HA doses described in Table 1. In contrast, ATZ and DEA commonly induced seven genes encoding regulatory and signaling proteins, representing $44 \%$ of ATZ-DEA commonly-induced genes (Figure 1, Supplemental Figure 1). ATZ specifically influenced expression of 8 genes of which 3 were repressed. DEA specifically induced expression of 10 genes while repressing only one. All loci showing common ATZ and DEA regulation, or specific regulation by either ATZ or DEA, were related either to abiotic stress adaptive responses or to stress hormone responses (Table 4). Low doses of ATZ and DEA were therefore perceived by plants as stress stimuli leading to stress-regulated gene activation. Among the three specifically ATZ-repressed genes, BHLH100, encoding a basic helix-loophelix transcription factor, is implicated in iron homeostasis [79]. While the two other ATZ- 
repressed genes are A-type Arabidopsis Response Regulator (ARR) genes, ARR6 and ARR7, which are negative regulators of CK signaling [81]. Interestingly independent studies [86,87] demonstrated that the Teosinte Branched1/Cycloidea/Proliferating cell factor20 (TCP20) protein, involved in regulation of cell division, cell growth and differentiation, regulates negatively BHLH100, ARR6 and ARR7. Furthermore, TCP20 is also known to directly regulate the LIPOXYGENASE2 ( $L O X 2)$ gene which encodes a chloroplast enzyme involved in jasmonic acid (JA) synthesis [88]. LOX2 was repressed after exposure to low doses of DEA (Supplemental Table 2) and also in conditions of high ATZ stress (Ma, mannitol $80 \mathrm{mM}$ plus atrazine $10 \mu \mathrm{M}$ ) and of ATZ tolerance (Sa, sucrose $80 \mathrm{mM}$ plus atrazine $10 \mu \mathrm{M}$ )[8]. This reinforces the suggestion of Dhaka et al. [89] that TCP transcription factors implicated in plant response to diverse environmental factors appears to play central role in signaling networks by interacting with many molecular, signaling and hormonal components. ATZ specifically induced three TF-encoding genes (Table 4), ERF17, SIG5 and HSFA2, a member of Heat Stress TF (Hsf) family, that were all, directly or indirectly, involved in chloroplast and photosynthesis protection under stress conditions (Table 4). Conversely, the stress-dependent accumulation of SIG5 transcripts is known to be suppressed by exogenous cytokinin in young plantlets [90]. These three stress-related markers responded to triazines independently from any impact on PSII, NPQ, respiration or root growth [4]. Plant response to low doses of triazines could therefore be construed as a response to triazine perception rather than to triazine-related perturbations or injuries.

Shared-function interaction analysis was carried out to identify potential relationships between triazine-regulated signaling proteins, pre-existing signaling systems and interaction networks implicated in the signaling of low dose responses. The thirty-one DEGs implicated in regulation and signaling were mapped (Figure 3) onto the STRING v.10 global Arabidopsis network [16]. Figure 3 focuses on differentially expressed genes, and therefore on transcript-level regulated genes. Connections with proteins that are regulated through posttranslational processes [91] could lead to identification of sensing processes.

Only five loci, NAP, BT4, ATL78, AOC2 and BHLH1OO, were not associated with any signaling network (Figure 3). Nearly all triazine-responsive signaling genes (Table 4) showed strong patterns of interaction (Figure 3), thus showing that triazine-regulated signaling was strongly coordinated. Among the five identified clusters, the most important consisted of 18 closely interconnected TFs whose expression was influenced by ATZ and/or DEA. The second network grouped two DEA- and/or ATZ-induced genes, whereas two other clusters grouped, 
respectively, two DEA-induced genes and two ATZ-repressed genes. ATZ and DEA therefore affected signaling networks that were clearly distinct from the HA-signaling network.

\subsection{Triazine-regulated networks of stress and environmental signaling}

The network containing the largest number of signaling component interactions strikingly revealed coordinated involvement of stress-, drought- and cold-response signaling genes in triazine responses (Figure 3). In this remarkable network, interconnected genes responded to ATZ, to DEA, or to both, thus suggesting that ATZ and DEA sensing, whether transcriptional or post-translational, converged on the same network.

Six TF-encoding genes in this network belong to APETALA2/ethylene-responsive element binding factor (AP2/ERF) family and are involved in plant abiotic stress responses [92]. DDF1, $C B F 1, C B F 2$ and ERF17 belong to Dehydration-Responsive Element Binding-protein (DREB) subfamily. RRTF1 and ERF105 are grouped under the ERF (ethylene response factor) subfamily B-3 of AP2/ERF involved in JA responses. DDF1, which is induced by both ATZ and DEA, is involved in tolerance to cold, drought, salinity and heat stresses in a hormonaldependent manner (Table 4). Magome et al. [45] suggested that, under high-salinity stress, DDF1 activates a gibberellin (GA) 2-oxidase, thus reducing endogenous GA levels and leading to reduced growth and stress adaptation. Both ATZ and DEA induced the transcriptional activator CBF1, which binds to DRE/CRT regulatory elements and induces COR (coldregulated) gene expression, thus leading to freezing tolerance. In contrast, $C B F 2$, involved in negative regulatory or feedback circuits of CBF pathway, was only induced by DEA. Both belong to the CBF regulon that acts like $D D F 1$ on plant stress responses. Salinity stress adaptation can be achieved by positive effects of $C B F$ genes on accumulation of DELLA proteins, which, as negative regulators of GA signaling, decrease GA action [48].

In parallel with coordinated involvement of cold- and salinity-stress-related genes, a small signaling component interaction network reflected heat-stress-related signaling processes. This network included two genes induced by DEA and/or ATZ (Figure 3), HSP17.6A, which encodes a HSP20-like chaperone superfamily protein, and HSFA2. HSFA2, a Heat Shock Factor, belongs to the heat shock response pathway as a transcriptional relay, and is involved in developmental processes and in acclimation to abiotic stresses (Table 4). HSP20-like proteins exhibit chaperone activity and are involved in stress tolerance (Table 4). Moreover, HSP20-like proteins are potentially regulated by HSFA2 during heat stress [93], thus suggesting combined 
roles in regulation of plant responses to low doses of triazines. HSFA2 and HSP17.6A are both related to protein folding surveillance and more particularly to responses to misfolded protein accumulation in cytosol during heat stress [94]. Interestingly, during heat shock, HSFA2 can also activate HSP21, a chloroplast heat-shock protein involved in core subunits of PSII protection from thermal damage through GUN5-dependent retrograde signaling pathway [95]. Involvement of surveillance systems under conditions of no or low toxicity [4] likely reflects sensing of discrete triazine-dependent perturbations.

Induction by DEA revealed involvement of circadian clock component PRR7 (Figure 3). PRR7 is a transcriptional repressor of regulators involved in plant growth, light signaling and stress responses and a modulator of ABA-regulated gene expression [63]. It is directly involved in circadian clock regulation through signal transduction mechanisms (Table 4). PRR7 takes part in oxidative stress responses and regulation of stomata opening, and may regulate cold and drought responses in coordination with ABA-dependent pathways [63]. While PRR7 protein represses during the day the morning phased CCA1 gene, the cell growth regulator TCP20 protein acts as direct transcriptional activator on $C C A 1$ expression [96]. Moreover, several genes defined as PRR7 targets [63] were induced by triazine treatments: $L H C B 3, C B F 1, C B F 2$, $L N K 2$, encoding a regulator of the clock-controlled anthocyanin biosynthetic pathway, and ABA1, which is involved in ABA biosynthesis (Tables 2, 4, Supplemental Table 2). Involvement of circadian clock-related mechanisms in responses to low doses of triazines emphasizes the sensitivity of the circadian clock, which is affected by high doses of xenobiotics [97]. Other genes included in this interaction network were consistent with light- and circadian clock-related pathways. ATZ-induced SIG5 gene, which activates chloroplastic genes, such as $p s b D$ encoding PSII D2 [59], is regulated by abiotic stress and light signals (Table 4). Moreover, ATZ-induced BBX16 gene, which interacts with SIG5 (Figure 3), is a stress-responsive TF involved in light signal transduction pathways (Table 4).

In this largest network, transcriptional repressor WRKY4O was in a central position, with interactions with CBF1 and CBF2. This regulator, strongly induced by both ATZ and DEA (Tables 3A-4), plays central roles in abiotic and biotic stress responses (Table 4). WRKY40 shares common abiotic, biotic and chemical stress responses with the STZ (ZAT10) gene [50], which was induced by low doses of ATZ and DEA. Both WRKY4O and STZ genes are important for abiotic stress tolerance (Table 4). Moreover, STZ and DEA-induced gene CAFla, both interacting with WRKY40 (Figure 3), are part of the Plant Core Environmental Stress Response [53], which groups genes commonly and systemically coordinated during abiotic stresses (UV- 
B, osmotic, salt, cold, drought and wounding stress). Moreover, during plant-fungus interactions, WRKY40 directly regulates key regulatory genes, like EDS1 involved in defense signaling, JAZ8, a member of JA-signaling repressor JAZ family, and RRTF1 [52], which was induced by DEA. RRTF1 responds to light, DCMU and salinity stress, and is involved in agedependent and systemic stress signaling and in the regulation of redox homeostasis (Table 4).

Genes specifically induced by HA were associated in a specific signaling component interaction network consisting of $L R L 3$, positive regulator of root hair development, $N A C 015$, transcription activator of root cap differentiation, and RAP2.11 (ERF TF family) (Figure 3, Table 4). Among the 60 genes identified as targets of NAC factors [77], 15 correspond to triazine-sensitive genes (Supplemental Table 2). These triazine-induced genes encode myrosinases (BGLU34, $B G L U 35), 3$ LEA-related proteins, 2 mannose-binding lectin proteins, the ribonuclease RNS3, the TatD related Dnase, the GNS1/SUR4 membrane protein, a fatty acid desaturase and a cisprenyltransferase. RAP2.11 is involved in the regulation of many root-related genes linked to ROS production, phytohormone signaling and calcium signaling [78], and more generally to root growth regulation under low-potassium conditions. RAP2.11 is a transcriptional regulator of $H A K 5$, encoding a major transporter of $\mathrm{K}^{+}$in roots. Moreover, RAP2.11 is a stress and ABA down-regulated gene that is part of universal cell-level stress responses thus highlighting relationships between stress regulation and root-specific expression [50]. There were therefore direct links between regulatory effects of HA (Figure 3) and growth and development effects of HA on roots [4]. These effects occurred under conditions of very weak interactions with PSII or mitochondrial complexes and therefore in absence of toxic effects [4]. It was striking that three signaling genes induced by low doses of HA belonged to coordinated TFs that are induced during salt stress response in roots [98]. Low doses of HA may thus be perceived through mechanisms involved in salt stress responses, with links between perception of low doses of HA and sodium or potassium dynamics.

\subsection{Triazine-regulated networks of hormone signaling}

The central interacting component in the largest signaling network (Figure 3), transcription regulator WRKY40, is closely related to ABA signaling processes [51]. WRKY40 negatively and directly regulates plant responses to $\mathrm{ABA}$ by modulating genes associated with $\mathrm{ABA}$ signaling; like $A B I 5, A B I 3$ and $S T Z$ [51]. GUN5, a Mg-chelatase $\mathrm{H}$ subunit involved in conversion of chlorophyll precursor protoporphyrin IX to Mg-protoporphyrin IX and a 
candidate chloroplastic ABA receptor, directly interacts with WRKY40 to regulate expression of ABA-responsive genes [99].

Other regulatory genes associated to this signaling network reflected relationships between triazine responses and ABA signaling. RRTF1, ERF105, MYBL2 and ERF17, which are involved in stress responses, cell homeostasis, and anthocyanin biosynthesis, were related to various hormones, especially ABA and additionally cytokinin for ERF105 and MYBL2 (Table 4). Moreover, CIPK20 (or SNRK3.6) encoding Calcineurin B-like (CBL)-Interacting Protein Kinase 20, a positive regulator of ABA signaling [65], was strongly induced ( $>4$-fold) by DEA (Tables 3A-4), and to a lesser extent, by ATZ and HA (Table 3A). CBL proteins are calcium sensors that bind to regulatory domains of CIPK protein through calcium-dependent processes [69]. CBL-CIPK signaling pathway is involved in abiotic and biotic stress, nutrient and ROS signaling and developmental processes (Table 4). Moreover, genes encoding calcium-binding and ABA-related proteins, like AT4g27280, BT5, and RD2O (Table 4), were induced by low doses of triazines, thus suggesting involvement of calcium-related signal transduction pathways in low-dose triazine responses. Erinle et al. [100] recently showed that calcium could protect Pennisetum seedlings from atrazine injury through effects on membrane integrity and PSII stability that may involve calmodulin- or calcineurin-like transduction pathways.

MYBL2 negatively regulates anthocyanin biosynthesis by repressing the expression of genes as DFR and ANS (or $L D O X$ ), encoding, respectively, a dihydroflavonol 4-reductase and an anthocyanidin synthase, implicated in anthocyanin-containing compound biosynthesis [56]. Therefore, the repressive effects of DEA on the expression of ANS and DFR could therefore be a consequence of the induction of MYBL2 (Supplemental Table 2). The major signaling component interaction network was linked, through the MYBL2 interacting protein, to a smaller network consisting of ARR6 and ARR7. These two A-type ARR genes were repressed by ATZ. Such downregulation has been reported for ARR6 in an oxidative stress context [81]. Moreover, Huang et al. [82] demonstrated that cotyledon greening was controlled by repressive effects of $\mathrm{ABI} 4$, a component of ABA signaling pathway, on $A-A R R$ gene transcription, thus emphasizing the importance of $\mathrm{ABA}$ and $\mathrm{CK}$ crosstalk for plant growth under conditions of abiotic stress.

All these triazine-regulated signaling genes (Figure 3) were directly involved in regulation of stress perception and adaptation responses (Table 4). Relationships between triazines and stress responses, as discussed above at metabolic and functional levels, could be ascribed to mobilization of stress signaling processes. This implies that low doses of triazines were perceived and interpreted as stress-like cues. According to Hahn et al. [53], stress-responsive 
genes are SA-, JA- or ABA-responsive. This was the case for a significant number of triazineresponsive signaling genes, with strong relationships with $\mathrm{ABA}$ and additional links with $\mathrm{CKs}$ (Figure 3, Table 4). Characterization of triazine-responsive signaling genes and networks also gave potential insights into primary regulators (GUN5, ABI4, ABI5, SnRKs, CCA1, TCP20) that may be involved in early perception of low doses of triazines and into unexpected second messengers of triazine perception, such as calcium and potassium.

\subsection{Stress- and hormone-related cis elements in triazine-responsive genes}

Links between triazine molecular effects, stress responses and hormonal regulations highlighted by triazine-responsive interaction networks (Figure 3) were confirmed by detailed analysis of GO annotations. Seventeen \% (34 DEGs) of all differentially expressed genes could be classified as hormone-related genes, according to GO annotations in TAIR database and Arabidopsis Hormone Database (http://ahd.cbi.pku.edu.cn/). This is 5-fold higher than the $3.34 \%$ proportion of hormone-related genes in the whole genome.

Search for cis-element motif enrichments in triazine-regulated genes was performed on promoters of 198 DEGs (Table 5A). Analysis showed over-representation with low p-values for ABRE (ABA-responsive element)-like binding sites, G-box promoter motifs [light-response element (LRE)], MYB4 binding site motifs, RY-repeat promoter motifs and Evening Element promoter motifs. In the specific case of DEA-responsive genes, the GATA promoter motif was found to be overrepresented with a very low p-value. Other DEA-related overrepresented motifs included ABRE-like binding sites, G-box promoter motifs, I-box promoter motifs, DPBF1\&2 binding sites and RY-repeat promoter motifs. Similar motifs (except I-box and RY-repeat promoter motifs) were statistically overrepresented in ATZ-regulated gene promoters. In contrast, over-representation of specific promoter motifs for HA-regulated genes was exclusively related to RY-repeat promoter motifs.

Most of the motifs identified are related to stresses and hormones, and in particular to ABA regulations, such as ABRE-like binding sites, G-box sites, MYB4 binding sites, RY-repeat motif and DPBF1\&2 sites. GATA motifs are involved in light-dependent and nitrate-dependent control of transcription, in BR-signaling pathways, and in CK responses. I-box motif involved in light signaling is linked to regulation of many genes encoding chloroplast proteins, while RY-repeat promoter motifs interact synergistically with ABA-responsive motifs. 
Such analysis confirmed that responses to low doses of triazines involved coordinated signaling pathways related to stress responses, light and hormones, with potential antagonist roles for CK and ABA signaling [102]. Involvement of CK signaling was reminiscent of previouslydescribed relationships between xenobiotic impacts and hormone-crosstalk transduction pathways $[8,22,23,103]$ and especially potential relationships between ATZ-related and CKrelated signaling pathways $[97,104,105]$.

\subsection{Cytokinin-related regulations}

Promoters of CK-regulated genes possess $[\mathrm{A} / \mathrm{G}] \mathrm{GAT}[\mathrm{T} / \mathrm{C}] \mathrm{CK}$ response motifs (CRM), specifically enabling binding of type-B ARR proteins [106]. This motif was extended to AAGAT[T,C]TT in the case of ARR1 binding [106]. Moreover, Brenner and Schmülling [17] identified potential CK-responsive cis-elements consisting of $[\mathrm{ACT}][\mathrm{AG}][\mathrm{GT}] \mathrm{AT}[\mathrm{ACT}][\mathrm{CT}][\mathrm{ACGT}]$ octameric sequences derived from the consensus sequence.

Promoters of all triazine-responsive DEGs were investigated for the presence of these novel CK-responsive cis elements [17]. Two octameric sequences, AAGATCTT and TATATATA, were significantly overrepresented in DEGs resulting from triazine treatments (Table 5B). According to Arabidopsis Hormone Database 2.0 and Brenner and Schmülling [17], CKsensitive genes represent $12 \%$ of protein-coding genes in the whole genome (3244 among 27,655 genes). In comparison, 17 among 31 signaling-related DEGs involved in triazine responses (55\%) are potentially CK-sensitive. Moreover, among the 198 triazine-responsive DEGs, 88 (44\%) were potentially CK-sensitive (Supplemental Table 4). Enrichment in CKresponsive cis elements and identification of CK-regulated genes therefore pointed out to important roles of $\mathrm{CK}$ signaling in triazine responses.

The 88 CK-related triazine-responsive DEGs were mapped onto the STRING v.10 global Arabidopsis network in order to highlight putative signaling component interactions (Supplemental Figure 3). 71 genes were integrated into six individual and independent cytokinin-responsive component interaction networks (N1 to N6).

N1, N2 and N3 networks were each restricted to two genes. All of these 6 genes were associated with abiotic, and in particular temperature, stresses. This suggested that low doses of triazines 
may interfere with CK pathways involved in modulation of low and high temperature shocks [80,107], or directly with temperature-related perception and transduction [101].

The five genes of N4 network were induced by HA and were principally related to proteolysis. $X C P 1, X C P 2$ and PRR1 are involved in secondary cell wall synthesis in roots [108]. Similarly, in N5 network, nine HA-induced loci exhibited functions related to cell wall organization, development and root elongation. N4 and N5 networks were therefore in close agreement with the HA-dependent regulatory network involving RAP2.11, LRL3 and NACO15 (Figure 3) and with the effects of HA on root growth [97].

N6 network involved 145 interactions between 50 CK-sensitive DEGs (Supplemental Figure 3). A first group of ten genes, induced by ATZ, DEA and HA, included genes encoding LEArelated proteins or involved in glucosinolate metabolism (BGLU34, and At1g52050, Atlg52060, Atlg52070). The second group was linked to the first through a putative interaction between a LEA protein (At1g52050) and ASN1. It consisted mostly in ATZ- and/or DEAinduced genes that were related to metabolic processes, such as GAT1.2.1, THA1, GLYI7, and BCAT-2 involved, respectively, in glutamine, threonine, carbohydrate and BCAA metabolic processes. Such networks indicated that triazine effects on carbon, nitrogen and energy metabolic pathways could involve cytokinin-related regulations. This group interacted with a third set of nineteen genes induced by ATZ and/or DEA, whose functions are mainly related to transcription regulation. As discussed above (Figure 3, Table 4), these genes, including BT5, CIPK20, MYBL2, STZ, and WRKY40, are important players for integrating stress, hormonal and nutritional signals.

This last group also interacted, through MYBL2, with ARR6 and ARR7, which were strongly repressed by ATZ, and act as Type-A response regulators, involved in negative regulations of CK signaling [81]. Moreover, individual gene analysis (Table 3B) showed that, among various CK-signaling-related genes, $A R R 6, A R R 7$ and also the $C R F 6$ cytokinin response factor were strongly affected by triazine treatments. Induction of expression of ARR6, ARR7 and, additionally, CRF6 is normally achieved by interaction of CKs with their canonical receptors [109]. CRF6 downregulates directly the expression of ARR6 during oxidative stress [81]. Repression of ARR6, ARR7 and also CRF6 (Table 3B) in presence of low doses of triazines may therefore reflect interferences with CK receptors. Moreover, since low doses of triazines did not affect expression of $A H K 2, A H K 3$ and $A H K 4$, which encode CK receptors [90], interference of triazines with cytokinin signaling likely involved direct post-translational effects of triazines on CK receptors. Cortleven et al. [110] reported important roles of cytokinin 
signaling in chloroplast development through direct transcriptional CK-regulation of chlorophyll biosynthesis genes (HEMA1, GUN4, GUN5, CHLM). Under low-dose exposure, ATZ induced HEMA1 expression (Table 3A), thus showing complex interactions of triazines with cytokinin regulation. Type-A response regulators also play a role in ABA responses [80], and ABA represses $A R R$ expression through mechanisms linked to CK biosynthesis and signal transduction [82]. The last group of N6 network included genes mainly related to terpenoid biosynthesis and root development, with two loci induced by all triazines, and four DEArepressed loci

Analysis of CK-sensitive triazine-regulated genes therefore showed that CK signaling could play important roles in triazine interplay with energy-, temperature-, drought- and ABAsignaling. Triazines at low doses may directly interact with mechanisms, including CK receptors [90], directly regulating ARR6, ARR7 and CRF6 expression. Analysis of underlying mechanisms could contribute to explain negative impacts of CKs on stress tolerance [102] and to predict antagonistic or beneficial effects of low doses of triazines on efficiency of abiotic stress responses [97].

\subsection{Primary signaling networks and targets}

As shown in Figure 4, the study of putative interactions between triazine-regulated signaling proteins confirmed the implication of signaling cascades in low-dose triazine responses, thus establishing a sensor-regulator-actuator regulation network as established by Singh et al. [111]. Transcript-level adjustment involved actuators, like ASN1, LEA, LHCB and JAC, and regulators, like ARR6-ARR7, WRKY40, CIPK20, CBF and MYBL2 (Tables 2-4). Moreover, interaction analysis indicated close relationships with crosstalk and sensing components like ABI4, ABI5, circadian clock components, GUN5, CK receptors and SnRKs (Figure 3). Thus intriguingly, low doses of triazines causing no or weak toxic effects induced patterns of signaling responses (Figure 4) involving ABA and CK pathways that were similar to salt, drought and cold signaling [112,113].

There was wide overlapping between hormone-responsive and triazine-responsive genes, with a $44 \%$ proportion of CK-sensitive genes in triazine-responsive genes. Comparison of lowtriazine transcriptomes with published transcriptomes revealed similarities with transcriptomic responses of CPL4(CTD phosphatase-like 4) RNAi plants [114]. Among 198 triazine-responsive DEGs (Figure 1), 30 were found differentially expressed in CPL4 $4_{\text {RNAi }}$ plants (Supplemental Table 5). Most of upregulated genes in CPL4 $4_{\text {RNAi }}$ plants were downregulated by triazines, and 
vice versa. Co-regulation of major triazine-responsive DEGs, like BGLU34, BGLU35, MYBL2, ASN1, IAA29, BT5, ARR7, and GSTF12, also occurred under conditions of CPL4 depletion [114]. Moreover, CPL4 depletion mimics xenobiotic stress induction, thus suggesting links between CPL4 perturbation and xenobiotic stress [114]. As in the case of low-triazine exposure (Table 3), CRF6 and ARR7 were identified as regulators of CPL4 depletion.

The effects of ATZ and DEA on gene expression were highly correlated with regulation of ABA- and CK-sensitive genes and with stress-related TFs that are not known to interact directly or indirectly with D1, the canonical ATZ target in PSII. In the absence of any PSII inhibition, HA affected gene expression (Figures 1-2), signaling processes (Figure 3) and CK-related regulations (Supplemental Figure 3) in a stress- and hormone-dependent manner. Transcriptomic effects of low doses of triazines were therefore independent from canonical target. Commonalities of response regulations in CPL4-depleted plants, xenobiotic-treated plants and low-triazine-treated plants (Figure 4) may be the perception of threats to cellular homeostasis and activation of surveillance systems.

\section{Conclusions}

Canonical-target-independent regulations should contribute to explain how xenobiotics with various structures and modes of action converge on common perturbations of energy, carbon and nitrogen metabolisms [4,7,23,41,42]. Underlying mechanisms and primary targets of such non-canonical cross-xenobiotic regulations remain to be unraveled. These xenobiotic primary targets must be related to a nexus of energy, temperature, drought, ionic, ABA and CK signaling (Figure 3, Table 4). These targets could act as integral part of sensing mechanisms allowing the perception of common chemical structures, like N-heterocycles present in cytokinins, triazines and various sulfonylurea herbicides [104,114]. Interestingly, one of the few abiotic-stress sensors identified in Arabidopsis, the histidine kinase AHK1, acts through an ABA-dependent pathway and belongs to the same Histidine Kinase (AHK) family as CK receptors [115]. Characterization of these perception mechanisms using signaling mutants will reveal relationships between xenobiotics, energy sensing, stress sensing, ABA and CK. Complete identification of non-canonical xenobiotic targets and underlying signaling pathways in plants is essential to assess early impacts of low and realistically environmental contaminating doses and of herbicides and degradation products on sensitive crops, on natural plant communities, and on ecosystem functioning. 


\section{Conflict of Interest Statement}

The authors declare that the research was conducted in the absence of any commercial or financial relationships that could be construed as a potential conflict of interest.

\section{Author Contributions}

D. A., I. C., C. S. and G. G. conceived the study and designed experiments. D. A. and G. G. performed experiments, S. P. performed microarray experiments and statistical analysis for microarray data. D. A., I. C., C. S. and G. G. carried out analysis and interpretation of experimental data including bioinformatics analyses. The article, which all authors edited and approved, was written by D. A., I. C., C. S. and G. G.

\section{Funding}

The POPS platform benefits from the support of LabExSaclay Plant Sciences-SPS (ANR-10LABX-0040-SPS).

\section{Acknowledgments}

DA is supported by a doctoral scholarship from Ministère de l'Education nationale, de l'Enseignement supérieur et de la Recherche (France).

\section{References}

[1] A. Hildebrandt, S. Lacorte, D. Barceló, Occurrence and fate of organochlorinated pesticides and PAH in agricultural soils from the Ebro river basin, Arch. Environ. Contam. Toxicol. 57 (2009) 247-255. doi:10.1007/s00244-008-9260-0.

[2] S. Sura, M. Waiser, V. Tumber, A. Farenhorst, Effects of herbicide mixture on microbial communities in prairie wetland ecosystems: A whole wetland approach, Sci. Total Environ. 435-436 (2012) 34-43. doi:10.1016/j.scitotenv.2012.07.003.

[3] A.-A. Serra, D. Alberto, C. Sulmon, G. Gouesbet, I. Couée, Implications des communautés végétales péri-agricoles dans la dynamique environnementale des pollutions par les pesticides, Rev Ecol-Terre Vie. 71 (2016) 203-221. 
[4] D. Alberto, I. Couée, C. Sulmon, G. Gouesbet, Root-level exposure reveals multiple physiological toxicity of triazine xenobiotics in Arabidopsis thaliana, J. Plant Physiol. 212 (2017) 105-114. doi:10.1016/j.jplph.2017.01.013.

[5] N.D. Jablonowski, A. Schäffer, P. Burauel, Still present after all these years: persistence plus potential toxicity raise questions about the use of atrazine, Environ. Sci. Pollut. Res. 18 (2011) 328-331. doi:10.1007/s11356-010-0431-y.

[6] A.W. Rutherford, A. Krieger-Liszkay, Herbicide-induced oxidative stress in photosystem II, Trends Biochem. Sci. 26 (2001) 648-653. doi:10.1016/S0968-0004(01)01953-3.

[7] A.-A. Serra, A. Nuttens, V. Larvor, D. Renault, I. Couée, C. Sulmon, G. Gouesbet, Low environmentally relevant levels of bioactive xenobiotics and associated degradation products cause cryptic perturbations of metabolism and molecular stress responses in Arabidopsis thaliana, J. Exp. Bot. 64 (2013) 2753-2766. doi:10.1093/jxb/ert119.

[8] F. Ramel, C. Sulmon, F. Cabello-Hurtado, L. Taconnat, M.-L. Martin-Magniette, J.-P. Renou, A. El Amrani, I. Couée, G. Gouesbet, Genome-wide interacting effects of sucrose and herbicide-mediated stress in Arabidopsis thaliana: novel insights into atrazine toxicity and sucrose-induced tolerance, BMC Genomics. 8 (2007) 450. doi:10.1186/1471-2164-8-450.

[9] D.C. Boyes, A.M. Zayed, R. Ascenzi, A.J. McCaskill, N.E. Hoffman, K.R. Davis, J. Görlach, Growth stage -based phenotypic analysis of Arabidopsis: a model for high throughput functional genomics in plants, Plant Cell. 13 (2001) 1499-1510.

[10] C. Lurin, C. Andrés, S. Aubourg, M. Bellaoui, F. Bitton, C. Bruyère, M. Caboche, C. Debast, J. Gualberto, B. Hoffmann, A. Lecharny, M. Le Ret, M.-L. Martin-Magniette, H. Mireau, N. Peeters, J.-P. Renou, B. Szurek, L. Taconnat, I. Small, Genome-wide analysis of Arabidopsis pentatricopeptide repeat proteins reveals their essential role in organelle biogenesis, Plant Cell. 16 (2004) 2089-2103. doi:10.1105/tpc.104.022236.

[11] S. Dèrozier, F. Samson, J.-P. Tamby, C. Guichard, V. Brunaud, P. Grevet, S. Gagnot, P. Label, J.-C. Leplé, A. Lecharny, S. Aubourg, Exploration of plant genomes in the FLAGdb $^{++}$environment, Plant Methods. 7 (2011) 8. doi:10.1186/1746-4811-7-8.

[12] Y.H. Yang, S. Dudoit, P. Luu, D.M. Lin, V. Peng, J. Ngai, T.P. Speed, Normalization for cDNA microarray data: a robust composite method addressing single and multiple slide systematic variation, Nucleic Acids Res. 30 (2002) e15.

[13] G.K. Smyth, Linear models and empirical Bayes methods for assessing differential expression in microarray experiments, Stat. Appl. Genet. Mol. Biol. 3 (2004) 1-25. doi:10.2202/1544-6115.1027.

[14] J.D. Storey, R. Tibshirani, Statistical significance for genome-wide studies, Proc. Natl. Acad. Sci. 100 (2003) 9440-9445. doi:10.1073/pnas.1530509100.

[15] R Core Team, R: A language and environment for statistical computing, reference index version 2.2.1., in: Vienna, Austria, 2005.

[16] D. Szklarczyk, A. Franceschini, S. Wyder, K. Forslund, D. Heller, J. Huerta-Cepas, M. Simonovic, A. Roth, A. Santos, K.P. Tsafou, M. Kuhn, P. Bork, L.J. Jensen, C. von Mering, STRING v10: protein-protein interaction networks, integrated over the tree of life, Nucleic Acids Res. 43 (2015) D447-D452. doi:10.1093/nar/gku1003.

[17] W.G. Brenner, T. Schmülling, Summarizing and exploring data of a decade of cytokininrelated transcriptomics, Front. Plant Sci. 6 (2015). doi:10.3389/fpls.2015.00029.

[18] S. Gagnot, J.-P. Tamby, M.-L. Martin-Magniette, F. Bitton, L. Taconnat, S. Balzergue, S. Aubourg, J.-P. Renou, A. Lecharny, V. Brunaud, CATdb: a public access to Arabidopsis transcriptome data from the URGV-CATMA platform, Nucleic Acids Res. 36 (2007) D986-D990. doi:10.1093/nar/gkm757. 
[19] C. Yang, J. Liu, X. Dong, Z. Cai, W. Tian, X. Wang, Short-Term and Continuing Stresses Differentially Interplay with Multiple Hormones to Regulate Plant Survival and Growth, Mol. Plant. 7 (2014) 841-855. doi:10.1093/mp/ssu013.

[20] S.R. Baerson, A. Sánchez-Moreiras, N. Pedrol-Bonjoch, M. Schulz, I.A. Kagan, A.K. Agarwal, M.J. Reigosa, S.O. Duke, Detoxification and transcriptome response in Arabidopsis seedlings exposed to the allelochemical benzoxazolin-2(3H)-one, J. Biol. Chem. 280 (2005) 21867-21881. doi:10.1074/jbc.M500694200.

[21] F. Ramel, C. Sulmon, M. Bogard, I. Couée, G. Gouesbet, Differential patterns of reactive oxygen species and antioxidative mechanisms during atrazine injury and sucrose-induced tolerance in Arabidopsis thaliana plantlets, BMC Plant Biol. 9 (2009) 28. doi:10.1186/1471-2229-9-28.

[22] F. Ramel, C. Sulmon, A.-A. Serra, G. Gouesbet, I. Couée, Xenobiotic sensing and signalling in higher plants, J. Exp. Bot. 63 (2012) 3999-4014. doi:10.1093/jxb/ers102.

[23] D. Weisman, M. Alkio, A. Colón-Carmona, Transcriptional responses to polycyclic aromatic hydrocarbon-induced stress in Arabidopsis thaliana reveal the involvement of hormone and defense signaling pathways, BMC Plant Biol. 10 (2010) 59. doi:10.1186/1471-2229-10-59.

[24] M. Su, G. Huang, Q. Zhang, X. Wang, C. Li, Y. Tao, S. Zhang, J. Lai, C. Yang, Y. Wang, The LEA protein, ABR, is regulated by ABI5 and involved in dark-induced leaf senescence in Arabidopsis thaliana, Plant Sci. 247 (2016) 93-103. doi:10.1016/j.plantsci.2016.03.009.

[25] A.L. Saucedo, E.E. Hernández-Domínguez, L.A. de Luna-Valdez, A.A. Guevara-García, A. Escobedo-Moratilla, E. Bojorquéz-Velázquez, F. del Río-Portilla, D.A. FernándezVelasco, A.P. Barba de la Rosa, Insights on structure and function of a late embryogenesis abundant protein from Amaranthus cruentus: an intrinsically disordered protein involved in protection against desiccation, oxidant conditions, and osmotic stress, Front. Plant Sci. 8 (2017). doi:10.3389/fpls.2017.00497.

[26] M. Song, W. Xu, Y. Xiang, H. Jia, L. Zhang, Z. Ma, Association of jacalin-related lectins with wheat responses to stresses revealed by transcriptional profiling, Plant Mol. Biol. 84 (2014) 95-110. doi:10.1007/s11103-013-0121-5.

[27] T. Janowitz, I. Trompetter, M. Piotrowski, Evolution of nitrilases in glucosinolatecontaining plants, Phytochemistry. 70 (2009) 1680-1686. doi:10.1016/j.phytochem.2009.07.028.

[28] W.R. Chezem, N.K. Clay, Regulation of plant secondary metabolism and associated specialized cell development by MYBs and bHLHs, Phytochemistry. 131 (2016) 26-43. doi:10.1016/j.phytochem.2016.08.006.

[29] M. Böhmer, J.I. Schroeder, Quantitative transcriptomic analysis of abscisic acid-induced and reactive oxygen species-dependent expression changes and proteomic profiling in Arabidopsis suspension cells: ABA- and ROS-regulated expression changes, Plant J. 67 (2011) 105-118. doi:10.1111/j.1365-313X.2011.04579.x.

[30] S.U. Huh, S.-B. Lee, H.H. Kim, K.-H. Paek, ATAF2, a NAC transcription factor, binds to the promoter and regulates NIT2 gene expression involved in auxin biosynthesis, Mol. Cells. 34 (2012) 305-313. doi:10.1007/s10059-012-0122-2.

[31] K. Dietrich, F. Weltmeier, A. Ehlert, C. Weiste, M. Stahl, K. Harter, W. Dröge-Laser, Heterodimers of the Arabidopsis transcription factors bZIP1 and bZIP53 reprogram amino acid metabolism during low energy stress, Plant Cell. 23 (2011) 381-395. doi:10.1105/tpc. 110.075390 .

[32] A. Mustroph, S.C. Lee, T. Oosumi, M.E. Zanetti, H. Yang, K. Ma, A. Yaghoubi-Masihi, T. Fukao, J. Bailey-Serres, Cross-kingdom comparison of transcriptomic adjustments to 
low-oxygen stress highlights conserved and plant-specific responses, Plant Physiol. 152 (2010) 1484-1500. doi:10.1104/pp.109.151845.

[33] C. Peng, S. Uygun, S.-H. Shiu, R. Last L., The impact of the branched-chain ketoacid dehydrogenase complex on amino acid homeostasis in Arabidopsis, Plant Physiol. (2015) pp.00461.2015. doi:10.1104/pp.15.00461.

[34] F. Ramel, C. Sulmon, G. Gouesbet, I. Couée, Regulatory effects of atrazine differentially override sucrose repression of amino acid catabolism, Acta Physiol. Plant. 35 (2013) 2329-2337. doi:10.1007/s11738-013-1246-5.

[35] M.V. Pires, A.A. Pereira Júnior, D.B. Medeiros, D.M. Daloso, P.A. Pham, K.A. Barros, M.K.M. Engqvist, A. Florian, I. Krahnert, V.G. Maurino, W.L. Araújo, A.R. Fernie, The influence of alternative pathways of respiration that utilize branched-chain amino acids following water shortage in Arabidopsis: Influence of the ETF/ETFQO pathway under water stress, Plant Cell Environ. 39 (2016) 1304-1319. doi:10.1111/pce.12682.

[36] H.M. Lam, M.H. Hsieh, G. Coruzzi, Reciprocal regulation of distinct asparagine synthetase genes by light and metabolites in Arabidopsis thaliana, Plant J. Cell Mol. Biol. 16 (1998) 345-353.

[37] E. Baena-González, F. Rolland, J.M. Thevelein, J. Sheen, A central integrator of transcription networks in plant stress and energy signalling, Nature. 448 (2007) 938-942. doi:10.1038/nature06069.

[38] P. Coello, S.J. Hey, N.G. Halford, The sucrose non-fermenting-1-related (SnRK) family of protein kinases: potential for manipulation to improve stress tolerance and increase yield, J. Exp. Bot. 62 (2011) 883-893. doi:10.1093/jxb/erq331.

[39] R.A. Gutiérrez, T.L. Stokes, K. Thum, X. Xu, M. Obertello, M.S. Katari, M. Tanurdzic, A. Dean, D.C. Nero, C.R. McClung, G.M. Coruzzi, Systems approach identifies an organic nitrogen-responsive gene network that is regulated by the master clock control gene CCAl, Proc. Natl. Acad. Sci. 105 (2008) 4939-4944. doi:10.1073/pnas.0800211105.

[40] J. Shin, A. Sánchez-Villarreal, A.M. Davis, S. Du, K.W. Berendzen, C. Koncz, Z. Ding, C. Li, S.J. Davis, The metabolic sensor AKIN10 modulates the Arabidopsis circadian clock in a light-dependent manner, Plant Cell Environ. 40 (2017) 997-1008. doi:10.1111/pce.12903.

[41] A.-A. Serra, I. Couée, D. Renault, G. Gouesbet, C. Sulmon, Metabolic profiling of Lolium perenne shows functional integration of metabolic responses to diverse subtoxic conditions of chemical stress, J. Exp. Bot. 66 (2015) 1801-1816. doi:10.1093/jxb/eru518.

[42] A.-A. Serra, I. Couée, D. Heijnen, S. Michon-Coudouel, C. Sulmon, G. Gouesbet, Genome-wide transcriptional profiling and metabolic analysis uncover multiple molecular responses of the grass species Lolium perenne under low-intensity xenobiotic stress, Front. Plant Sci. 6 (2015). doi:10.3389/fpls.2015.01124.

[43] D.G. Hardie, F.A. Ross, S.A. Hawley, AMPK: a nutrient and energy sensor that maintains energy homeostasis, Nat. Rev. Mol. Cell Biol. 13 (2012) 251-262. doi:10.1038/nrm3311.

[44] W. Sun, C. Bernard, B. van de Cotte, M. Van Montagu, N. Verbruggen, At-HSP17.6A, encoding a small heat-shock protein in Arabidopsis, can enhance osmotolerance upon overexpression, Plant J. Cell Mol. Biol. 27 (2001) 407-415.

[45] H. Magome, S. Yamaguchi, A. Hanada, Y. Kamiya, K. Oda, The DDF1 transcriptional activator upregulates expression of a gibberellin-deactivating gene, GA2ox7, under highsalinity stress in Arabidopsis, Plant J. 56 (2008) 613-626. doi:10.1111/j.1365313X.2008.03627.x.

[46] H.-G. Kang, J. Kim, B. Kim, H. Jeong, S.H. Choi, E.K. Kim, H.-Y. Lee, P.O. Lim, Overexpression of $F T L 1 / D D F 1$, an AP2 transcription factor, enhances tolerance to cold, 
drought, and heat stresses in Arabidopsis thaliana, Plant Sci. 180 (2011) 634-641. doi:10.1016/j.plantsci.2011.01.002.

[47] C. Zhao, Z. Zhang, S. Xie, T. Si, Y. Li, J.-K. Zhu, Mutational evidence for the critical role of CBF transcription factors in cold acclimation in Arabidopsis, Plant Physiol. (2016) pp.00533.2016. doi:10.1104/pp.16.00533.

[48] M. Zhou, H. Chen, D. Wei, H. Ma, J. Lin, Arabidopsis CBF3 and DELLAs positively regulate each other in response to low temperature, Sci. Rep. 7 (2017) 39819. doi:10.1038/srep39819.

[49] H.-Y. Seok, D.-H. Woo, L.V. Nguyen, H.T. Tran, V.N. Tarte, S.M.M. Mehdi, S.-Y. Lee, Y.-H. Moon, Arabidopsis AtNAP functions as a negative regulator via repression of AREB1 in salt stress response, Planta. 245 (2017) 329-341. doi:10.1007/s00425-0162609-0.

[50] S. Ma, H.J. Bohnert, Integration of Arabidopsis thaliana stress-related transcript profiles, promoter structures, and cell-specific expression, Genome Biol. 8 (2007) R49. doi:10.1186/gb-2007-8-4-r49.

[51] H. Chen, Z. Lai, J. Shi, Y. Xiao, Z. Chen, X. Xu, Roles of Arabidopsis WRKY18, WRKY40 and WRKY60 transcription factors in plant responses to abscisic acid and abiotic stress, BMC Plant Biol. 10 (2010) 281. doi:10.1186/1471-2229-10-281.

[52] S.P. Pandey, M. Roccaro, M. Schön, E. Logemann, I.E. Somssich, Transcriptional reprogramming regulated by WRKY18 and WRKY40 facilitates powdery mildew infection of Arabidopsis: WRKY18/40 in powdery mildew susceptibility, Plant J. 64 (2010) 912-923. doi:10.1111/j.1365-313X.2010.04387.x.

[53] A. Hahn, J. Kilian, A. Mohrholz, F. Ladwig, F. Peschke, R. Dautel, K. Harter, K. Berendzen, D. Wanke, Plant core environmental stress response genes are systemically coordinated during abiotic stresses, Int. J. Mol. Sci. 14 (2013) 7617-7641. doi:10.3390/ijms14047617.

[54] D. Zhang, H. Ye, H. Guo, A. Johnson, H. Lin, Y. Yin, Transcription factors involved in brassinosteroid repressed gene expression and their regulation by BIN2 kinase, Plant Signal. Behav. 9 (2014) e27849.

[55] N.H. Nguyen, C.Y. Jeong, G.-H. Kang, S.-D. Yoo, S.-W. Hong, H. Lee, MYBD employed by HY5 increases anthocyanin accumulation via repression of MYBL2 in Arabidopsis, Plant J. 84 (2015) 1192-1205. doi:10.1111/tpj.13077.

[56] S. Kim, G. Hwang, S. Lee, J.-Y. Zhu, I. Paik, T.T. Nguyen, J. Kim, E. Oh, High Ambient Temperature Represses Anthocyanin Biosynthesis through Degradation of HY5, Front. Plant Sci. 8 (2017). doi:10.3389/fpls.2017.01787.

[57] P. Jacob, H. Hirt, A. Bendahmane, The heat-shock protein/chaperone network and multiple stress resistance, Plant Biotechnol. J. 15 (2017) 405-414. doi:10.1111/pbi.12659.

[58] A. Nagashima, M. Hanaoka, T. Shikanai, M. Fujiwara, K. Kanamaru, H. Takahashi, K. Tanaka, The multiple-stress responsive plastid sigma factor, SIG5, directs activation of the $p s b D$ blue light-responsive promoter (BLRP) in Arabidopsis thaliana, Plant Cell Physiol. 45 (2004) 357-368.

[59] M.V. Yamburenko, Y.O. Zubo, T. Börner, Abscisic acid affects transcription of chloroplast genes via protein phosphatase $2 \mathrm{C}$-dependent activation of nuclear genes: repression by guanosine-3'-5'-bisdiphosphate and activation by sigma factor 5, Plant $\mathrm{J}$. 82 (2015) 1030-1041. doi:10.1111/tpj.12876.

[60] X. Yin, X. Xie, X. Xia, J. Yu, I.B. Ferguson, J.J. Giovannoni, K. Chen, Involvement of an ethylene response factor in chlorophyll degradation during citrus fruit degreening, Plant J. 86 (2016) 403-412. doi:10.1111/tpj.13178. 
[61] N.H. Davila Olivas, W. Kruijer, G. Gort, C.L. Wijnen, J.J.A. van Loon, M. Dicke, Genome-wide association analysis reveals distinct genetic architectures for single and combined stress responses in Arabidopsis thaliana, New Phytol. 213 (2017) 838-851. doi:10.1111/nph.14165.

[62] Z. Zhang, R. Ji, H. Li, T. Zhao, J. Liu, C. Lin, B. Liu, CONSTANS-LIKE 7 (COL7) is involved in phytochrome B (phyB)-mediated light-quality regulation of auxin homeostasis, Mol. Plant. 7 (2014) 1429-1440. doi:10.1093/mp/ssu058.

[63] T. Liu, J. Carlsson, T. Takeuchi, L. Newton, E.M. Farré, Direct regulation of abiotic responses by the Arabidopsis circadian clock component PRR7, Plant J. (2013) n/a-n/a. doi:10.1111/tpj.12276.

[64] E. Kolmos, B.Y. Chow, J.L. Pruneda-Paz, S.A. Kay, HsfB2b-mediated repression of PRR7 directs abiotic stress responses of the circadian clock, Proc. Natl. Acad. Sci. 111 (2014) 16172-16177. doi:10.1073/pnas.1418483111.

[65] D. Gong, C. Zhang, X. Chen, Z. Gong, J.-K. Zhu, Constitutive activation and transgenic evaluation of the function of an Arabidopsis PKS protein kinase, J. Biol. Chem. 277 (2002) 42088-42096. doi:10.1074/jbc.M205504200.

[66] P. Kanwar, S.K. Sanyal, I. Tokas, A.K. Yadav, A. Pandey, S. Kapoor, G.K. Pandey, Comprehensive structural, interaction and expression analysis of CBL and CIPK complement during abiotic stresses and development in rice, Cell Calcium. 56 (2014) 8195. doi:10.1016/j.ceca.2014.05.003.

[67] J.W. Walley, D.R. Kelley, G. Nestorova, D.L. Hirschberg, K. Dehesh, Arabidopsis deadenylases AtCAF1a and AtCAF1b play overlapping and distinct roles in mediating environmental stress responses, Plant Physiol. 152 (2010) 866-875. doi:10.1104/pp.109.149005.

[68] S. Bolt, E. Zuther, S. Zintl, D.K. Hincha, T. Schmülling, ERF105 is a transcription factor gene of Arabidopsis thaliana required for freezing tolerance and cold acclimation: ERF105 and cold stress, Plant Cell Environ. 40 (2017) 108-120. doi:10.1111/pce.12838.

[69] M. Matsuo, J.M. Johnson, A. Hieno, M. Tokizawa, M. Nomoto, Y. Tada, R. Godfrey, J. Obokata, I. Sherameti, Y.Y. Yamamoto, F.-D. Böhmer, R. Oelmüller, High REDOX RESPONSIVE TRANSCRIPTION FACTOR1 Levels Result in Accumulation of Reactive Oxygen Species in Arabidopsis thaliana Shoots and Roots, Mol. Plant. 8 (2015) 1253-1273. doi:10.1016/j.molp.2015.03.011.

[70] A. Bahieldin, A. Atef, S. Edris, N.O. Gadalla, H.M. Ali, S.M. Hassan, M.A. Al-Kordy, A.M. Ramadan, R.M. Makki, A.S.M. Al-Hajar, F.M. El-Domyati, Ethylene responsive transcription factor ERF109 retards PCD and improves salt tolerance in plant, BMC Plant Biol. 16 (2016). doi:10.1186/s12870-016-0908-z.

[71] C. Gleason, R.C. Foley, K.B. Singh, Mutant analysis in Arabidopsis provides insight into the molecular mode of action of the auxinic herbicide dicamba, PLoS ONE. 6 (2011) e17245. doi:10.1371/journal.pone.0017245.

[72] M.A. Koini, L. Alvey, T. Allen, C.A. Tilley, N.P. Harberd, G.C. Whitelam, K.A. Franklin, High temperature-mediated adaptations in plant architecture require the bHLH transcription factor PIF4, Curr. Biol. 19 (2009) 408-413. doi:10.1016/j.cub.2009.01.046.

[73] L. Du, B.W. Poovaiah, A novel family of $\mathrm{Ca}^{2+} /$ calmodulin-binding proteins involved in transcriptional regulation: interaction with fsh/Ring3 class transcription activators, Plant Mol. Biol. 54 (2004) 549-569. doi:10.1023/B:PLAN.0000038269.98972.bb.

[74] H.S. Robert, A. Quint, D. Brand, A. Vivian-Smith, R. Offringa, BTB and TAZ domain scaffold proteins perform a crucial function in Arabidopsis development: Arabidopsis BT proteins and gametophyte development, Plant J. 58 (2009) 109-121. doi:10.1111/j.1365313X.2008.03764.X. 
[75] S. Bogamuwa, J.-C. Jang, The Arabidopsis tandem CCCH zinc finger proteins AtTZF4, 5 and 6 are involved in light-, abscisic acid- and gibberellic acid-mediated regulation of seed germination: Tandem $\mathrm{CCCH}$ zinc finger proteins in seed germination, Plant Cell Environ. 36 (2013) 1507-1519. doi:10.1111/pce.12084.

[76] T.H.Y. Tam, B. Catarino, L. Dolan, Conserved regulatory mechanism controls the development of cells with rooting functions in land plants, Proc. Natl. Acad. Sci. 112 (2015) E3959-E3968. doi:10.1073/pnas.1416324112.

[77] M. Kamiya, S.-Y. Higashio, A. Isomoto, J.-M. Kim, M. Seki, S. Miyashima, K. Nakajima, Control of root cap maturation and cell detachment by BEARSKIN transcription factors in Arabidopsis, Development. 143 (2016) 4063-4072. doi:10.1242/dev.142331.

[78] M.J. Kim, D. Ruzicka, R. Shin, D.P. Schachtman, The Arabidopsis AP2/ERF transcription factor RAP2.11 modulates plant response to low-potassium conditions, Mol. Plant. 5 (2012) 1042-1057. doi:10.1093/mp/sss003.

[79] M. Martínez-Trujillo, A. Méndez-Bravo, R. Ortiz-Castro, F. Hernández-Madrigal, E. Ibarra-Laclette, L.F. Ruiz-Herrera, T.A. Long, C. Cervantes, L. Herrera-Estrella, J. López-Bucio, Chromate alters root system architecture and activates expression of genes involved in iron homeostasis and signaling in Arabidopsis thaliana, Plant Mol. Biol. 86 (2014) 35-50. doi:10.1007/s11103-014-0210-0.

[80] J. Jeon, N.Y. Kim, S. Kim, N.Y. Kang, O. Novák, S.-J. Ku, C. Cho, D.J. Lee, E.-J. Lee, M. Strnad, J. Kim, A subset of cytokinin two-component signaling system plays a role in cold temperature stress response in Arabidopsis, J. Biol. Chem. 285 (2010) 23371-23386. doi:10.1074/jbc.M109.096644.

[81] P.J. Zwack, I. De Clercq, T.C. Howton, H.T. Hallmark, A. Hurny, E.A. Keshishian, A.M. Parish, E. Benkova, M.S. Mukhtar, F. Van Breusegem, A.M. Rashotte, Cytokinin Response Factor 6 represses cytokinin-associated genes during oxidative stress, Plant Physiol. (2016) pp.00415.2016. doi:10.1104/pp.16.00415.

[82] X. Huang, X. Zhang, Z. Gong, S. Yang, Y. Shi, ABI4 represses the expression of type-A $A R R s$ to inhibit seed germination in Arabidopsis, Plant J. 89 (2017) 354-365. doi:10.1111/tpj.13389.

[83] S.J. Kim, W.T. Kim, Suppression of Arabidopsis RING E3 ubiquitin ligase AtATL78 increases tolerance to cold stress and decreases tolerance to drought stress, FEBS Lett. 587 (2013) 2584-2590. doi:10.1016/j.febslet.2013.06.038.

[84] J.Y. Suh, S.J. Kim, T.R. Oh, S.K. Cho, S.W. Yang, W.T. Kim, Arabidopsis Tóxicos en Levadura 78 (AtATL78) mediates ABA-dependent ROS signaling in response to drought stress, Biochem. Biophys. Res. Commun. 469 (2016) 8-14. doi:10.1016/j.bbrc.2015.11.061.

[85] P. Ahmad, S. Rasool, A. Gul, S.A. Sheikh, N.A. Akram, M. Ashraf, A.M. Kazi, S. Gucel, Jasmonates: multifunctional roles in stress tolerance, Front. Plant Sci. 7 (2016). doi:10.3389/fpls.2016.00813.

[86] C. Herve, P. Dabos, C. Bardet, A. Jauneau, M.C. Auriac, A. Ramboer, F. Lacout, D. Tremousaygue, In vivo interference with AtTCP20 function induces severe plant growth alterations and deregulates the expression of many genes important for development, Plant Physiol. 149 (2009) 1462-1477. doi:10.1104/pp.108.126136.

[87] M.E. Andriankaja, S. Danisman, L.F. Mignolet-Spruyt, H. Claeys, I. Kochanke, M. Vermeersch, L. De Milde, S. De Bodt, V. Storme, A. Skirycz, F. Maurer, P. Bauer, P. Mühlenbock, F. Van Breusegem, G.C. Angenent, R.G.H. Immink, D. Inzé, Transcriptional coordination between leaf cell differentiation and chloroplast development established by TCP20 and the subgroup Ib bHLH transcription factors, Plant Mol. Biol. 85 (2014) 233-245. doi:10.1007/s11103-014-0180-2. 
[88] S. Danisman, F. van der Wal, S. Dhondt, R. Waites, S. de Folter, A. Bimbo, A.D. van Dijk, J.M. Muino, L. Cutri, M.C. Dornelas, G.C. Angenent, R.G.H. Immink, Arabidopsis class I and class II TCP transcription factors regulate jasmonic acid metabolism and leaf development antagonistically, Plant Physiol. 159 (2012) 1511-1523. doi:10.1104/pp.112.200303.

[89] N. Dhaka, V. Bhardwaj, M.K. Sharma, R. Sharma, Evolving tale of TCPs: new paradigms and old lacunae, Front. Plant Sci. 8 (2017). doi:10.3389/fpls.2017.00479.

[90] M.N. Danilova, N.V. Kudryakova, A.S. Doroshenko, D.A. Zabrodin, Z.F. Rakhmankulova, R. Oelmüller, V.V. Kusnetsov, Opposite roles of the Arabidopsis cytokinin receptors AHK2 and AHK3 in the expression of plastid genes and genes for the plastid transcriptional machinery during senescence, Plant Mol. Biol. 93 (2017) 533-546. doi:10.1007/s11103-016-0580-6.

[91] C. Merchante, A.N. Stepanova, J.M. Alonso, Translation regulation in plants: an interesting past, an exciting present and a promising future, Plant J. 90 (2017) 628-653. doi:10.1111/tpj.13520.

[92] J. Mizoi, K. Shinozaki, K. Yamaguchi-Shinozaki, AP2/ERF family transcription factors in plant abiotic stress responses, Biochim. Biophys. Acta BBA - Gene Regul. Mech. 1819 (2012) 86-96. doi:10.1016/j.bbagrm.2011.08.004.

[93] S. Kotak, E. Vierling, H. Baumlein, P. v. Koskull-Doring, A novel transcriptional cascade regulating expression of heat stress proteins during seed development of Arabidopsis, Plant Cell Online. 19 (2007) 182-195. doi:10.1105/tpc.106.048165.

[94] D.H. Kim, Z.-Y. Xu, Y.J. Na, Y.-J. Yoo, J. Lee, E.-J. Sohn, I. Hwang, Small heat shock protein Hsp17.8 functions as an AKR2A cofactor in the targeting of chloroplast outer membrane proteins in Arabidopsis, Plant Physiol. 157 (2011) 132-146. doi:10.1104/pp.111.178681.

[95] S.-T. Chen, N.-Y. He, J.-H. Chen, F.-Q. Guo, Identification of core subunits of photosystem II as action sites of HSP21, which is activated by the GUN5-mediated retrograde pathway in Arabidopsis, Plant J. 89 (2017) 1106-1118. doi:10.1111/tpj.13447.

[96] J.-F. Wu, H.-L. Tsai, I. Joanito, Y.-C. Wu, C.-W. Chang, Y.-H. Li, Y. Wang, J.C. Hong, J.-W. Chu, C.-P. Hsu, S.-H. Wu, LWD-TCP complex activates the morning gene CCA1 in Arabidopsis, Nat. Commun. 7 (2016) 13181. doi:10.1038/ncomms 13181.

[97] D. Alberto, A.-A. Serra, C. Sulmon, G. Gouesbet, I. Couée, Herbicide-related signaling in plants reveals novel insights for herbicide use strategies, environmental risk assessment and global change assessment challenges, Sci. Total Environ. 569-570 (2016) 16181628. doi:10.1016/j.scitotenv.2016.06.064.

[98] J. Nie, R. Stewart, H. Zhang, J.A. Thomson, F. Ruan, X. Cui, H. Wei, TF-Cluster: A pipeline for identifying functionally coordinated transcription factors via network decomposition of the shared coexpression connectivity matrix (SCCM), BMC Syst. Biol. 5 (2011) 53. doi:10.1186/1752-0509-5-53.

[99] O. Van Aken, B. Zhang, S. Law, R. Narsai, J. Whelan, AtWRKY40 and AtWRKY63 Modulate the Expression of Stress-Responsive Nuclear Genes Encoding Mitochondrial and Chloroplast Proteins, Plant Physiol. $162 \quad$ (2013) 254-271. doi:10.1104/pp.113.215996.

[100] K.O. Erinle, Z. Jiang, B. Ma, J. Li, Y. Chen, K. Ur-Rehman, A. Shahla, Y. Zhang, Exogenous calcium induces tolerance to atrazine stress in Pennisetum seedlings and promotes photosynthetic activity, antioxidant enzymes and $p s b A$ gene transcripts, Ecotoxicol. Environ. Saf. 132 (2016) 403-412. doi:10.1016/j.ecoenv.2016.06.035.

[101] K. Shinozaki, K. Yamaguchi-Shinozaki, Molecular responses to dehydration and low temperature: differences and cross-talk between two stress signaling pathways, Curr. Opin. Plant Biol. 3 (2000) 217-223. 
[102] P.J. Zwack, A.M. Rashotte, Interactions between cytokinin signalling and abiotic stress responses, J. Exp. Bot. 66 (2015) 4863-4871. doi:10.1093/jxb/erv172.

[103] C. Sulmon, G. Gouesbet, A. El Amrani, I. Couée, Involvement of the ethylene-signalling pathway in sugar-induced tolerance to the herbicide atrazine in Arabidopsis thaliana seedlings, J. Plant Physiol. 164 (2007) 1083-1092. doi:10.1016/j.jplph.2006.11.005.

[104] I. Couée, A.-A. Serra, F. Ramel, G. Gouesbet, C. Sulmon, Physiology and toxicology of hormone-disrupting chemicals in higher plants, Plant Cell Rep. 32 (2013) 933-941. doi:10.1007/s00299-013-1428-z.

[105] A.-A. Serra, D. Alberto, F. Ramel, G. Gouesbet, C. Sulmon, I. Couée, Perturbation and disruption of plant hormone signalling by organic xenobiotic pollutions, in: G.K. Pandey (Ed.), Mech. Plant Horm. Signal. Stress, John Wiley \& Sons, Inc., Hoboken, NJ, USA, 2017: pp. 199-221. doi:10.1002/9781118889022.ch25.

[106] E. Ramireddy, W.G. Brenner, A. Pfeifer, A. Heyl, T. Schmülling, In Planta Analysis of a cis-Regulatory Cytokinin Response Motif in Arabidopsis and Identification of a Novel Enhancer Sequence, Plant Cell Physiol. 54 (2013) 1079-1092. doi:10.1093/pcp/pct060.

[107] M. Cerný, P.L. Jedelský, J. Novák, A. Schlosser, B. Brzobohatý, Cytokinin modulates proteomic, transcriptomic and growth responses to temperature shocks in Arabidopsis: Cytokinin modulates temperature-shock responses, Plant Cell Environ. 37 (2014) 16411655. doi:10.1111/pce.12270.

[108] R.A. Chávez Montes, G. Coello, K.L. González-Aguilera, N. Marsch-Martínez, S. de Folter, E.R. Alvarez-Buylla, ARACNe-based inference, using curated microarray data, of Arabidopsis thaliana root transcriptional regulatory networks, BMC Plant Biol. 14 (2014) 97. doi:10.1186/1471-2229-14-97.

[109] P.J. Zwack, B.R. Robinson, M.G. Risley, A.M. Rashotte, Cytokinin response factor 6 negatively regulates leaf senescence and is induced in response to cytokinin and numerous abiotic stresses, Plant Cell Physiol. 54 (2013) 971-981. doi:10.1093/pcp/pct049.

[110] A. Cortleven, I. Marg, M.V. Yamburenko, H. Schlicke, K. Hill, B. Grimm, G.E. Schaller, T. Schmülling, Cytokinin regulates the etioplast-chloroplast transition through the twocomponent signaling system and activation of chloroplast-related genes, Plant Physiol. 172 (2016) 464-478. doi:10.1104/pp.16.00640.

[111] A.H. Singh, D.M. Wolf, P. Wang, A.P. Arkin, Modularity of stress response evolution, Proc. Natl. Acad. Sci. U. S. A. 105 (2008) 7500-7505. doi:10.1073/pnas.0709764105.

[112] I. Hwang, J. Sheen, B. Müller, Cytokinin signaling networks, Annu. Rev. Plant Biol. 63 (2012) 353-380. doi:10.1146/annurev-arplant-042811-105503.

[113] P.E. Verslues, ABA and cytokinins: challenge and opportunity for plant stress research, Plant Molecular Biology. 91 (2016) 629-640. doi:10.1007/s11103-016-0458-7.

[114] A. Fukudome, E. Aksoy, X. Wu, K. Kumar, I.S. Jeong, K. May, W.K. Russell, H. Koiwa, Arabidopsis CPL4 is an essential C-terminal domain phosphatase that suppresses xenobiotic stress responses, Plant J. 80 (2014) 27-39. doi:10.1111/tpj.12612.

[115] Y. Osakabe, K. Yamaguchi-Shinozaki, K. Shinozaki, L.-S.P. Tran, Sensing the environment: key roles of membrane-localized kinases in plant perception and response to abiotic stress, J. Exp. Bot. 64 (2013) 445-458. doi:10.1093/jxb/ers354.

\section{Legends of figures}

Figure 1 
Distribution and overlap of triazine-responsive differentially expressed genes. Venn diagrams of triazine-responsive DEGs. Arabidopsis plantlets were grown on Hoagland medium during thirteen days and then transferred during 24 hours on Hoagland media containing $1 \mu \mathrm{M}$ ATZ, DEA or HA. A statistical cut-off ( $p$-value $\leq 0.05$ after Bonferroni correction and $\mid \log _{2}$ (ratio) $\mid>1$ ) was used to define the genes that were significantly differentially expressed (DEGs) in comparison with control.

\section{Figure 2}

Hierarchical clustering and heatmap representation of the expression profiles of triazineresponsive DEGs. The hierarchical clustering representation was generated using Cluster 3.0 software (http://bonsai.hgc.jp/ mdehoon/software/cluster) and Java Tree View (http://jtreeview.sourceforge.net). The sample clustering tree appears at the right, the DEG clustering tree appears at the top. Color key gives the level of expression of genes differentially expressed and not differentially expressed (NDE, $\mid \log _{2}($ ratio $\left.) \mid<1\right)$.

\section{Figure 3}

Shared-function networks of triazine-responsive DEGs involved in signaling and transcription. Shared-function interaction networks for the 31 DEGs encoding signaling components and transcription factors (TFs) were mapped onto STRING v.10 global Arabidopsis networks [16]. Pink and blue areas include, respectively, induced genes and repressed genes in a given network. Letters in the circles associated with each gene show patterns of triazine responses: A, induced by ATZ; D, induced by DEA and A/D induced by ATZ and DEA. Line thickness indicates the strength of data support. Arabidopsis Genome Initiative (AGI) codes and corresponding gene names are given in Supplemental Table 2.

\section{Figure 4}

Integrative scheme of triazine-related signaling. The different components of plant responses to low doses of triazines are shown as sensors, regulators and actuators [111]. Positive and negative responses to low doses of triazines are respectively indicated in red and blue. 


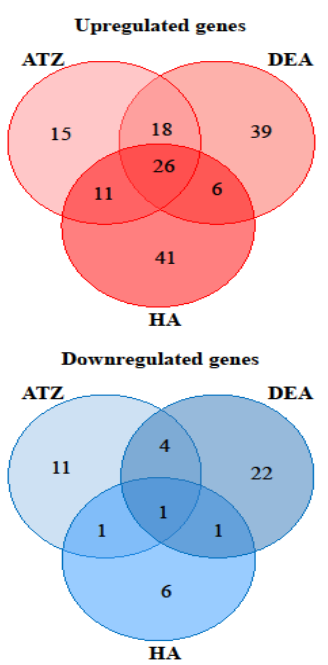

Figure 1

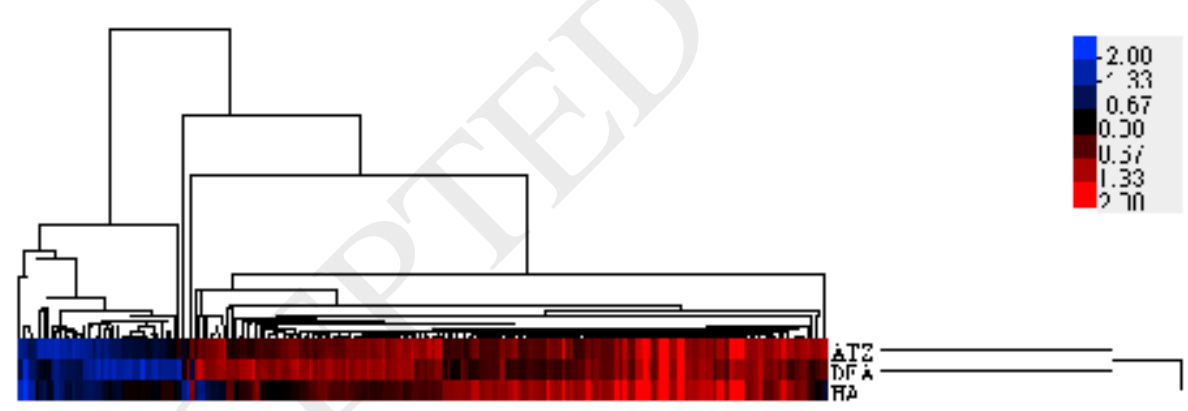

Figure 2 

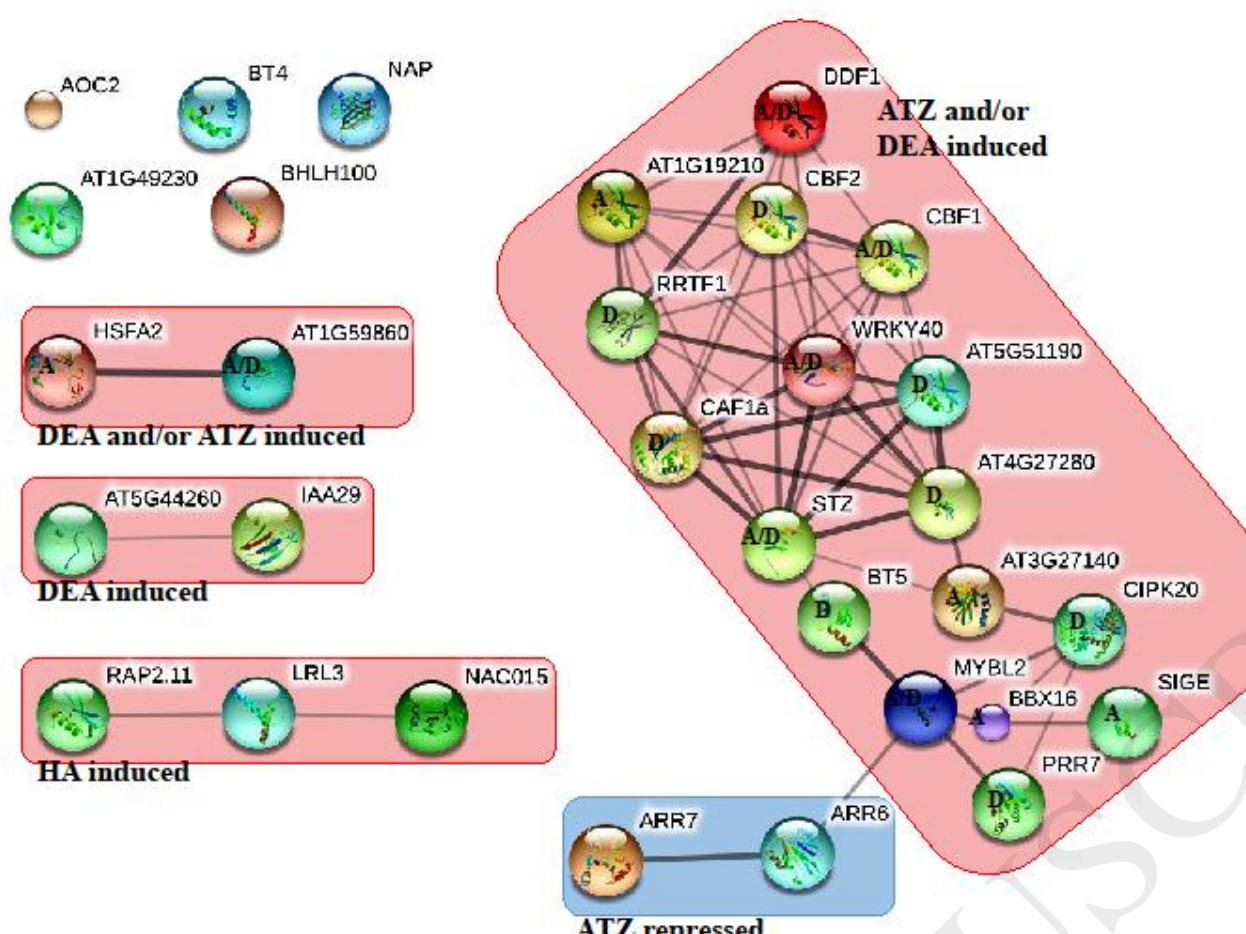

ATZ repressed

Figure 3

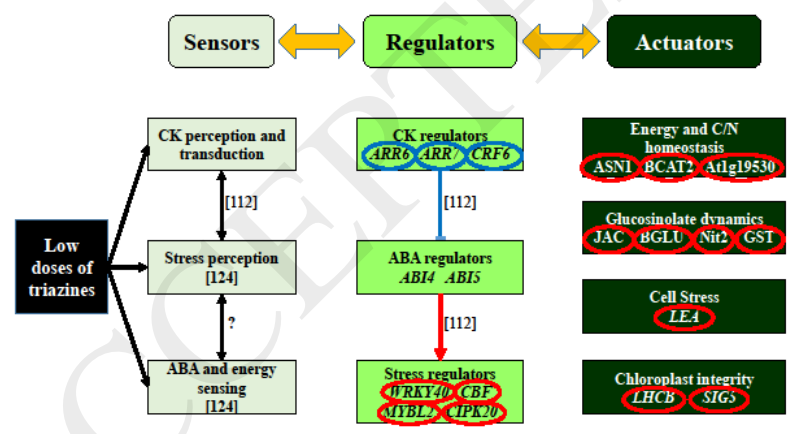

Figure 4 
Table 1. Significantly-enriched GO Slim terms in triazine-responsive genes

\begin{tabular}{|c|c|c|c|c|c|c|}
\hline & \multicolumn{6}{|c|}{ Normed Frequency } \\
\hline & \multicolumn{2}{|c|}{ ATZ } & \multicolumn{2}{|c|}{ DEA } & \multicolumn{2}{|c|}{ HA } \\
\hline & Induced & Repressed & Induced & Repressed & Induced & Repressed \\
\hline \multicolumn{7}{|l|}{ Biological process } \\
\hline developmental processes & & & & & $2.26^{* * *}$ & \\
\hline other biological processes & $1.61^{*}$ & $3.74 * *$ & $2.63 * * *$ & $3.29 * *$ & & $3.32 *$ \\
\hline other cellular processes & $1.36^{*}$ & & $1.26^{*}$ & & $1.29 *$ & \\
\hline protein metabolism & & & $0.15^{* * *}$ & & $0.35^{*}$ & \\
\hline response to abiotic or biotic stimulus & $2.64 * * *$ & & $2.97 * * *$ & $2.69^{*}$ & & $3.32 *$ \\
\hline response to stress & $2.32^{* * *}$ & $2.56^{*}$ & $2.36^{* * *}$ & $2.01 *$ & & $3.19 *$ \\
\hline unknown biological processes & $0.37 * * *$ & & $0.43^{* *}$ & $0.27 *$ & $0.47 * *$ & \\
\hline \multicolumn{7}{|l|}{ Molecular function } \\
\hline hydrolase activity & $2.07 *$ & & $1.95^{*}$ & & $2.91 * * *$ & \\
\hline other enzyme activity & $2.81 * *$ & & $1.73^{*}$ & $4.87 * *$ & $2.47^{*}$ & \\
\hline other molecular functions & & $11.75^{*}$ & $3.11^{*}$ & & & \\
\hline transcription factor activity & $2.59^{*}$ & & $2.1^{*}$ & & & \\
\hline unknown molecular functions & $0.54 *$ & & & $0.14 *$ & $0.75^{*}$ & \\
\hline \multicolumn{7}{|l|}{ Cellular component } \\
\hline cell wall & $2.96^{*}$ & $10.32 *$ & & & $3.61 *$ & \\
\hline extracellular & $2.93 * * *$ & & $1.93 *$ & $2.40^{*}$ & $4.51 * * *$ & $4.46^{*}$ \\
\hline mitochondria & & & & & $0.29 *$ & \\
\hline plasma membrane & $0.13 *$ & & $0.39 *$ & & & \\
\hline
\end{tabular}

ATZ: atrazine; DEA: desethylatrazine; HA: hydroxyatrazine. Only significantly over-represented and underrepresented GO Slim terms are shown. They were determined by Classification SuperViewer (http://bar.utoronto.ca/) with normalized class score option. Significantly-enriched GO Slim terms with pvalue $<0.01,0.001$ and 0.0001 were indicated by one, two and three asterisks, respectively. Shading indicates those categories that show more than 2-fold $(>2)$ overrepresentation or less than two-fold $(<0.5)$ underrepresentation. Supplemental Table 3 gave for each set of genes classical GO annotations, namely GO terms, and corresponding broader category-termed GO Slim. 
Table 2. Comparative effects of low and high doses of triazines on transcriptomic responses of stress- and metabolism-related genes

\begin{tabular}{llccccc} 
& & \multicolumn{4}{c}{ Log $_{2}$ (ratio)* } \\
\cline { 3 - 6 } AGI & Description & ATZ & DEA & HA & Ma & Sa \\
\hline AT3G19430 & Late embryogenesis abundant (LEA) protein-related & 1.51 & 1.38 & 2.48 & NDE & NDE \\
AT5G54370 & Late embryogenesis abundant (LEA) protein-related & 1.61 & 1.42 & 2.41 & NDE & NDE \\
AT5G60530 & Late embryogenesis abundant (LEA) protein-related & 1.11 & 1.20 & 1.54 & NDE & NDE \\
AT1G47600 & BGLU34, Beta Glucosidase 34 & 3.79 & 3.31 & 4.16 & NP & NP \\
AT1G51490 & BGLU36, Beta Glucosidase 36 & 3.52 & 3.10 & 3.96 & NP & NP \\
AT1G51470 & BGLU35, Beta Glucosidase 35 & 2.78 & 2.50 & 3.40 & NP & NP \\
AT1G52050 & Mannose-binding lectin superfamily protein & 2.69 & 2.42 & 3.48 & NDE & NDE \\
AT1G52060 & Mannose-binding lectin superfamily protein & 1.21 & 1.19 & 2.04 & NDE & NDE \\
AT3G44300 & NIT2, Nitrilase 2 & NDE & 1.03 & NDE & 1.01 & 0.83 \\
AT4G35770 & DIN1, SEN1, Senescence 1 & NDE & 1.25 & NDE & 1.05 & 3.14 \\
AT3G47340 & ASN1, Glutamine-Dependent Asparagine Synthase 1 & 1.20 & 1.35 & NDE & 1.52 & 3.90 \\
AT4G03060 & AOP2, Alkenyl Hydroxalkyl Producing 2 & NDE & -1.09 & NDE & -2.06 & -1.99 \\
AT1G78370 & GSTU20, Glutathione S-Transferase Tau 20 & NDE & -1.12 & NDE & -3.72 & -2.13 \\
AT5G17220 & GSTF12, Glutathione S-Transferase Phi 12 & NDE & -1.69 & NDE & -1.52 & -3.12 \\
AT1G10070 & BCAT2, Branched-Chain Amino Acid Transaminase 2 & NDE & 1.11 & -1.03 & 1.09 & 2.65 \\
AT1G19530 & unknown protein involved in low-oxygen response & NDE & 1.61 & -1.05 & 3.64 & 4.47 \\
AT5G54270 & LHCB3, Light-Harvesting Chlorophyll B-Binding 3 & 1.10 & 1.20 & 1.38 & -0.83 & -1.10 \\
AT3G47470 & LHCA4, Light-Harvesting Chlorophyll-Protein & NDE & 1.00 & 1.13 & -0.99 & NDE \\
& Complex I Subunit A4 & & & & & \\
\hline
\end{tabular}

*CATMAv7 data are expressed by normalized $\log _{2}$ (ratio) generated by statistical analysis of relative expression between treatment and control. A statistical cut-off ( $\mathrm{p}$-value $\leq 0.05$ after Bonferroni correction and $\mid \log 2($ ratio) $\mid>1$ ) was used to determine which genes were significantly differentially expressed in comparison with control. ATZ: atrazine; DEA: desethylatrazine; HA: hydroxyatrazine. Complementary gene expression data were extracted from transcriptomic profiling experiments registered as E-MEXP-411 in ArrayExpress [8] with Ma [toxic treatment with $10 \mu \mathrm{M}$ ATZ in the absence of exogenous sucrose and in the presence of $80 \mathrm{mM}$ mannitol as osmotic control] and $\mathrm{Sa}$ [stress tolerance treatment with $10 \mu \mathrm{M}$ ATZ in the presence of $80 \mathrm{mM}$ exogenous sucrose)] comparisons. Differentially expressed genes were genes showing at least one $\mathrm{P}$ value $<0.05$ after Bonferroni correction, in one of the Ma or Sa comparisons [8]. NDE: not differentially expressed, NP: not present in array. 
Table 3. Individual gene qRT-PCR analysis of triazine effects on expression of molecular markers

A- Expression dynamics [ $\log _{2}($ ratio)] of major triazine-regulated molecular markers

\begin{tabular}{llccc}
\hline AGI & Genes & ATZ & DEA & HA \\
\hline AT1G52050 & JAC & $4.89 \pm 0.55$ & $3.40 \pm 0.88$ & $5.24 \pm 0.22$ \\
AT5G54370 & LEA & $2.82 \pm 0.56$ & $3.51 \pm 0.95$ & $0.73 \pm 1.64$ \\
AT1G80840 & WRKY40 & $1.98 \pm 0.82$ & $1.54 \pm 0.42$ & $0.48 \pm 0.06$ \\
AT1G71030 & MYBL2 & $1.81 \pm 0.34$ & $1.509 \pm 0.34$ & $1.28 \pm 0.64$ \\
AT1G10070 & BCAT2 & $1.68 \pm 0.17$ & $1.34 \pm 0.02$ & $-0.47 \pm 0.09$ \\
AT1G58290 & HEMA1 & $1.44 \pm 0.02$ & $0.35 \pm 0.04$ & $0.87 \pm 0.23$ \\
AT5G45820 & CIPK20 & $1.10 \pm 0.20$ & $2.11 \pm 0.53$ & $1.13 \pm 0.07$ \\
AT3G47340 & ASN1 & $1.01 \pm 0.06$ & $1.84 \pm 0.58$ & $-0.03 \pm 1.04$ \\
\hline
\end{tabular}

B- Expression dynamics [ $\log _{2}($ ratio)] of genes linked to cytokinin signaling

\begin{tabular}{llccc}
\hline AGI & Genes & ATZ & DEA & HA \\
\hline AT5G35750 & AHK2 & $0.56 \pm 0.29$ & $0.08 \pm 0.00$ & $0.55 \pm 0.28$ \\
AT1G27320 & AHK3 & $0.32 \pm 0.06$ & $0.45 \pm 0.11$ & $0.28 \pm 0.20$ \\
AT2G01830 & AHK4 & $-0.33 \pm 0.15$ & $-0.51 \pm 0.34$ & $0.02 \pm 0.15$ \\
AT3G61630 & CRF6 & $-0.74 \pm 0.24$ & $-0.33 \pm 0.15$ & $-0.21 \pm 0.04$ \\
AT1G19050 & ARR7 & $-1.20 \pm 0.49$ & $-1.10 \pm 0.28$ & $-0.39 \pm 0.07$ \\
AT5G62920 & ARR6 & $-1.19 \pm 0.25$ & $-0.54 \pm 0.10$ & $-1.70 \pm 0.68$ \\
\hline
\end{tabular}

Changes in expression levels were expressed as $\log _{2}($ ratio) relative to the control samples normalized to Protein Phosphatase $2 A(P P 2 A)$ gene expression. The qRT-PCR values were in general agreement with microarray data. ATZ: atrazine; DEA: desethylatrazine; HA: hydroxyatrazine. Data represents the average of three independent biological samples and two technical replicates and standard error of mean $( \pm \mathrm{SEM})$. 
Table 4. Expression patterns of triazine-responsive genes involved in signaling and gene regulation processes

\begin{tabular}{|c|c|c|c|c|c|c|}
\hline \multirow[b]{2}{*}{ AGI } & \multirow[b]{2}{*}{ Protein name } & \multicolumn{3}{|c|}{$\log _{2}$ (ratio)* } & \multirow[t]{2}{*}{ Typical physiological involvement } & \multirow[t]{2}{*}{ Reference } \\
\hline & & ATZ & DEA & HA & & \\
\hline AT1G59860 & HSP17.6A & 1.66 & 1.08 & NDE & Heat, drought, osmolarity, stress tolerance & [44] \\
\hline AT1G12610 & DDF1 & 1.53 & 1.35 & NDE & Cold, drought, heat, salinity, stress tolerance, GA & {$[45,46]$} \\
\hline AT4G25490 & CBF1 & 1.32 & 1.29 & NDE & Salinity, cold, GA, JA, ABA, ethylene, stress tolerance, development & {$[47,48]$} \\
\hline AT1G69490 & NAP, ANAC029 & 1.32 & 1.58 & NDE & Drought, salinity, ABA & [49] \\
\hline AT1G80840 & WRKY40 & 1.20 & 1.20 & NDE & Drought, salinity, osmotic, pathogen, ABA, cold, osmolarity, wounding, stress signaling & {$[50-52]$} \\
\hline AT1G27730 & STZ, ZAT10 & 1.06 & 1.30 & NDE & Cold, salinity, UV-B, osmolarity, wounding, pathogen, drought, stress tolerance & {$[50,53]$} \\
\hline AT1G71030 & MYBL2 & 1.03 & 1.38 & NDE & Light, brassinosteroids, heat, cytokinin, anthocyanin biosynthesis & {$[54-56]$} \\
\hline AT2G26150 & HSFA2 & 1.35 & NDE & NDE & $\begin{array}{l}\text { Heat, anoxia, salinity, osmolarity, oxidative stress, PSII protection, development, stress } \\
\text { resistance }\end{array}$ & [57] \\
\hline AT3G27140 & Protein phosphatase $2 \mathrm{C}$ & 1.12 & NDE & NDE & Cold, osmotic, salinity, wounding, pathogen, UV-B, drought, stress signaling & {$[50,53]$} \\
\hline AT5G24120 & SIG5, SIGE & 1.09 & NDE & NDE & Light, cold, salinity, osmolarity, ABA, stress protection & {$[58,59]$} \\
\hline AT1G19210 & ERF17 & 1.08 & NDE & NDE & Pathogen, drought, JA, ABA, ethylene, chlorophyll degradation & {$[60,61]$} \\
\hline AT1G73870 & BBX16, COL7 & 1.07 & NDE & NDE & Light, auxin, light signal perception & [62] \\
\hline AT5G02810 & PRR7 & NDE & 1.61 & NDE & Salinity, heat, oxidative stress, circadian clock, ABA, stomata opening, stress response & {$[63,64]$} \\
\hline AT4G25470 & CBF2 & NDE & 1.39 & NDE & Cold, salinity, GA, JA, ethylene, stress tolerance, development & {$[47,48]$} \\
\hline AT5G45820 & CIPK20 & NDE & 1.39 & NDE & ABA, calcium signaling, stress, nutrient and ROS signaling, development & {$[65,66]$} \\
\hline AT3G44260 & CAF1a & NDE & 1.30 & NDE & $\begin{array}{l}\text { Wounding, JA, ABA, SA, pathogen, salinity, UV-B, osmolarity, cold, drought, stress } \\
\text { tolerance }\end{array}$ & {$[53,67]$} \\
\hline AT5G51190 & ERF105 & NDE & 1.28 & NDE & $\begin{array}{l}\text { Light, cold, osmolarity, salinity, wounding, pathogen, ABA, JA, cytokinin, ethylene, } \\
\text { gibberellins, brassinolides, stress tolerance }\end{array}$ & {$[50,68]$} \\
\hline AT4G34410 & RRTF1, ERF109 & NDE & 1.23 & NDE & $\begin{array}{l}\text { Light, ethylene, ABA, JA, methyl viologen, drought, cold, UV-B, heat, osmotic, salinity } \\
\text { stress, pathogen, redox homeostasis, stress tolerance, lateral root formation, programmed } \\
\text { cell death and environmental signal transduction }\end{array}$ & {$[69,70]$} \\
\hline AT4G27280 & $\begin{array}{l}\text { Calcium-binding EF-hand } \\
\text { protein }\end{array}$ & NDE & 1.18 & NDE & Calcium, dicamba, cold, osmolarity, salinity, wounding, pathogen, stress responsive & {$[50,71]$} \\
\hline AT4G32280 & IAA29 & NDE & 1.12 & NDE & Dicamba, auxin, light, heat & {$[71,72]$} \\
\hline AT4G37610 & BT5 & NDE & 1.07 & NDE & Calcium, cold, $\mathrm{H}_{2} \mathrm{O}_{2}, \mathrm{SA}$, auxin, development, organic nitrogen & {$[39,73,74]$} \\
\hline AT5G44260 & TZF5 & NDE & 1.06 & NDE & $\mathrm{ABA}, \mathrm{GA}$, drought, light, stress response & [75] \\
\hline AT5G58010 & LRL3 & NDE & NDE & 1.15 & Auxin, root development & [76] \\
\hline AT1G33280 & NAC015, BEARSKIN 1 & NDE & NDE & 1.11 & Root cap differentiation & [87] \\
\hline AT5G19790 & RAP2.11 & NDE & NDE & 1.03 & Cold, osmotic, salinity, drought, ethylene, ABA, JA, low-potassium, root growth & {$[50,78]$} \\
\hline AT5G67480 & BT4 & NDE & NDE & -1.26 & Calcium, $\mathrm{H}_{2} \mathrm{O}_{2}$, salinity, wounding, JA, SA, auxin, development & {$[73,74]$} \\
\hline AT2G41240 & BHLH100 & -1.08 & NDE & NDE & Root iron homeostasis, chromate & [79] \\
\hline
\end{tabular}




\begin{tabular}{lllllll} 
AT5G62920 & ARR6 & -1.26 & NDE & NDE & Cytokinin signaling, cold, ABA, $\mathrm{H}_{2} \mathrm{O}_{2}$ \\
AT1G19050 & ARR7 & -1.45 & NDE & NDE & Cytokinin signaling, cold, ABA \\
AT1G49230 & ATL78 & NDE & -1.05 & NDE & ROS-mediated ABA signaling, drought, cold, salinity, protein degradation \\
AT3G25770 & AOC2 & -1.06 & -1.42 & NDE & JA biosynthesis, stress tolerance, development \\
\hline
\end{tabular}

*CATMA data are expressed by normalized $\log _{2}$ (ratio) generated by statistical analysis of relative expression between treatment and control. ATZ: atrazine; DEA: desethylatrazine; HA: hydroxyatrazine. A statistical cut-off ( $p$-value $\leq 0.05$ after Bonferroni correction and $\mid \log 2$ (ratio) $\mid>1$ ) was used to determine which genes were significantly differentially expressed in comparison with the control. Annotations were obtained on TAIR10 database. NDE: not differentially expressed. Data represents the average of two independent biological samples and two technical replicates 
Table 5. Identification of enriched promoter motifs in triazine-regulated genes

\section{A- Identification of enriched promoter motifs}

\begin{tabular}{|c|c|c|c|c|}
\hline & \multicolumn{3}{|l|}{ Motif } & p-value \\
\hline & \multicolumn{3}{|c|}{ ABRE-like binding site } & $2.34 \mathrm{E}-13$ \\
\hline & \multicolumn{3}{|c|}{ G-box promoter motif [LRE] } & $2.15 \mathrm{E}-07$ \\
\hline \multirow[t]{3}{*}{ All DEGs } & \multicolumn{3}{|c|}{ MYB4 binding site motif } & $2.66 \mathrm{E}-05$ \\
\hline & \multicolumn{3}{|c|}{ RY-repeat promoter motif } & $3.22 \mathrm{E}-04$ \\
\hline & \multicolumn{3}{|c|}{ Evening Element promoter motif } & 4.69E-04 \\
\hline \multirow{4}{*}{ ATZ-regulated } & \multicolumn{3}{|c|}{ DPBF1\&2 binding site motif } & $6.90 \mathrm{E}-06$ \\
\hline & \multicolumn{3}{|c|}{ ABRE-like binding site motif } & $3.47 \mathrm{E}-05$ \\
\hline & \multicolumn{3}{|c|}{ G-box promoter motif [LRE] } & 7.00E-04 \\
\hline & \multicolumn{3}{|c|}{ GATA promoter motif [LRE] } & 6.84E-04 \\
\hline \multirow{6}{*}{ DEA-regulated } & \multicolumn{3}{|c|}{ GATA promoter motif [LRE] } & $1.75 \mathrm{E}-26$ \\
\hline & \multicolumn{3}{|c|}{ ABRE-like binding site motif } & 5.31E-11 \\
\hline & \multicolumn{3}{|c|}{ G-box promoter motif [LRE] } & 2.85E-08 \\
\hline & \multicolumn{3}{|c|}{ I-box promoter motif } & 2.10E-04 \\
\hline & \multicolumn{3}{|c|}{ DPBF1\&2 binding site motif } & 5.15E-04 \\
\hline & \multicolumn{3}{|c|}{ RY-repeat promoter motif } & 5.60E-04 \\
\hline HA-regulated & \multicolumn{3}{|c|}{ RY-repeat promoter motif } & 7.22E-05 \\
\hline \multicolumn{5}{|c|}{ Enrichment in CK-responsive cis-elements } \\
\hline & & $\mathrm{M}$ & & \\
\hline & & TCTT & & TATA \\
\hline & Enrichment & $\mathrm{p}$-value & Enrichment & $\mathrm{p}$-value \\
\hline All DEGs & 3.38 & $7.00 \mathrm{E}-04$ & 2.04 & $1.08 \mathrm{E}-02$ \\
\hline ATZ-regulated & 4.34 & $1.87 \mathrm{E}-02$ & 2.10 & 4.97E-02 \\
\hline DEA-regulated & 2.24 & $2.47 \mathrm{E}-02$ & 2.09 & $2.70 \mathrm{E}-02$ \\
\hline HA-regulated & 2.31 & $2.49 \mathrm{E}-02$ & 2.15 & $2.15 \mathrm{E}-02$ \\
\hline
\end{tabular}

Identification of enriched promoter motifs was undertaken with Arabidopsis promoter motif search programs (http://stan.cropsci.uiuc.edu/cgi-bin/elefinder/compare.cgi). Overrepresentation of CK-responsive cis-elements was estimated as described by Brenner and Schmülling [17]. Motifs in promoters were counted using Promomer (http://bbc.botany.utoronto.ca/ntools/cgi-bin/BAR_Promomer.cgi) and significantly enriched motifs for DEG promoters were calculated against a control set of least likely CK-induced genes [17]. ATZ: atrazine; DEA: desethylatrazine; HA: hydroxyatrazine. 